\title{
Exosome-loaded conductive hydrogel with immune- modulating and neurogenesis-enhancing properties for synergistic spinal cord injury repair
}

\section{Lei Fan}

South China University of Technology

Can Liu

The Third Affiliated Hospital of Sun Yat-sen University

\section{Xiuxing Chen}

Sun Yat-sen University Cancer Center

\section{Yan Zou}

Third Affiliated Hospital of Sun Yat-Sen University

\section{Huiquan Wen}

Third Affiliated Hospital of Sun Yat-Sen University

\section{Fang Lu}

Beijing University of Chinese Medicine

\section{Yian Luo}

Guangdong University of Technology

\section{Guoxin Tan}

Guangdong University of Technology

\section{Peng Yu}

South China University of Technology https://orcid.org/0000-0002-2253-7373

\section{Dafu Chen}

Beijing Research Institute of Traumatology and Orthopaedics, Beijing Jishuitan Hospital

\section{Qiyou Wang}

the Third Affiliated Hospital of Sun Yat-sen University

\section{Lei Zhou}

South China University of Technology

Chengyun Ning ( $\square$ imcyning@scut.edu.cn )

South China University of Technology

\section{Article}

Keywords: Spinal cord injury, Conductive hydrogels, BMSCs, Exosomes, miRNAs, Anti-inflammation, Neurogenesis, Axonal regeneration 
Posted Date: September 29th, 2020

DOl: https://doi.org/10.21203/rs.3.rs-69851/v1

License: (c) (i) This work is licensed under a Creative Commons Attribution 4.0 International License. Read Full License 
1 Exosome-loaded conductive hydrogel with immune-

Lei $\mathrm{Fan}^{1,2 \dagger}{ }^{2 \dagger}$ Can $\mathrm{Liu}^{2,3 \dagger}$, Xiuxing $\mathrm{Chen}^{4 \dagger}$, Yan Zou ${ }^{6}$, Huiquan Wen ${ }^{6}$, Fang Lu ${ }^{8}$, Yian Luo ${ }^{5}$, Guoxin Tan ${ }^{5}$, Peng Yu ${ }^{1}$, Dafu Chen ${ }^{7}$, Qiyou Wang ${ }^{2 *}$, Lei Zhou ${ }^{1 *}$, Chenyun Ning ${ }^{1 *}$

\author{
Affiliations \\ ${ }^{1}$ School of Materials Science and Engineering \& National Engineering Research Center for \\ Tissue Restoration and Reconstruction, South China University of Technology, Guangzhou \\ 510641, China \\ ${ }^{2}$ Department of Spine Surgery, the Third Affiliated Hospital of Sun Yat-sen University, \\ Guangzhou 510630, China. \\ ${ }^{3}$ Department of Spine Surgery, the First Hospital of Zhejiang University, Hangzhou 310003, \\ China. \\ ${ }^{4}$ State Key Laboratory of Oncology in South China, Sun Yat-sen University Cancer Center, \\ Guangzhou 510630, China. \\ ${ }^{5}$ School of Chemical Engineering and Light Industry, Guangdong University of Technology, \\ Guangzhou 510006, China. \\ ${ }^{6}$ Department of Radiology, the Third Affiliated Hospital of Sun Yat-Sen University, Guangzhou \\ 510630, China. \\ ${ }^{7}$ Laboratory of Bone Tissue Engineering, Beijing Research Institute of Orthopaedics and \\ Traumatology, Beijing JiShuiTan Hospital, Beijing 100035, China \\ ${ }^{8}$ School of Preclinical Medicine, Beijing University of Chinese Medicine, Beijing 100029, China \\ $\dagger$ The first three authors contributed equally to this work \\ *Corresponding author. Email: imcyning@scut.edu.cn or ning_lab@hotmail.com (C.N.); zhoul@ \\ scut.edu.cn (L.Z.); wqiyou@163.com (Q.W.)
}
Abstract
Conductive hydrogels are very attractive candidates for accelerated spinal cord injury (SCI) repair because they match the electrical and mechanical properties of neural tissue. However, conductive hydrogel implantation can potentially aggravate inflammation, and hinder its repair efficacy. Bone marrow stem cell-derived exosomes (BMSC-exos) have shown immunomodulatory and tissue regeneration effects, therefore, we developed neural tissue-like conductive hydrogels loaded with BMSC-exos for the synergistic treatment of SCI. These exos- loaded conductive hydrogels modulated microglial M2 polarization via the $\mathrm{NF}-\kappa \mathrm{B}$ pathway, and synergistically enhanced neuronal and oligodendrocyte differentiation of neural stem cells (NSCs) while inhibiting astrocyte differentiation, and also increased axon outgrowth via the PTEN/PI3K/AKT/mTOR pathway. Furthermore, exos combined conductive hydrogels significantly decreased the number of CD68-positive microglia, enhanced local neurogenesis, and promoted axonal regeneration, resulting in significant functional recovery at the early stage in an SCI mouse model. Hence, the findings of this study demonstrate that the combination of conductive hydrogels and BMSC-exos is a promising therapeutic strategy for SCI repair. 
Key word: Spinal cord injury, Conductive hydrogels, BMSCs, Exosomes, miRNAs, Antiinflammation, Neurogenesis, Axonal regeneration

\section{Introduction}

An estimated 27 million people live with long-term disability following spinal cord injury (SCI) worldwide, with approximately 180,000 new cases occurring each year [1]. SCI is followed by neuronal loss and axon and myelin necrosis, which leads to an extensive inflammatory response and further exacerbates secondary damage [2]. Meanwhile, activated inflammatory cells (microglia) release pro-inflammatory cytokines, which contribute to reactive astrocyte gathering, subsequently increasing the release of inhibitory molecules such as chondroitin sulfate proteoglycans (CSPGs) at the injury site [3]. Because of the inflammatory microenvironment and limited neural regeneration capacity, the injury is resolved by the formation of a dense glial scar, which acts as a barrier to neuron and axon regeneration [4]. Thus, modulation of the inflammatory microenvironment, enhancement of local neurogenesis, and guidance and promotion of axon growth are needed for treating SCI.

Decompressive surgery with re-establishment of spinal stability and high-dose intravenous methylprednisolone sodium succinate (MPSS) usage in the acute phase of injury ( $\leq 8$ hours) are the most common clinical treatments at present [5]. However, the former only aims to avoid further secondary damage by relieving the pressure on the injured spinal cord, and the latter can reduce early inflammatory responses but with severe complications, while neither show the ability to promote axon and neuron regeneration and therefore, have limited therapeutic effectiveness. In this regard, experimental approaches to promote axon growth including cellular transplantation and scaffold biomaterials have been applied in SCI repair [6]. Cellular transplantation has been used experimentally in clinical conditions with some success but continues to be limited by uncontrolled cell differentiation, low survival rates, ethical issues, and the inevitable cell loss after implantation [7]. Considering the seriousness of these problems, cost-effective and cell-free biomaterial implants are highly desirable.

Scaffold biomaterial-based therapy has been proposed as a strategy to promote neural tissue regeneration by providing $3 \mathrm{D}$ matrices with the desired biological, chemical, and physical characteristics that favor cellular attachment, growth, differentiation, and neurite extension [8, 9]. In particular, since the soft and hydrated forms of hydrogels are similar to native nerve tissue, they are widely used to promote cellular growth and tissue formation after SCI [7, 10, 11]. Currently, conductive hydrogels have emerged as a promising class of hydrogel scaffolds combining a hydrophilic matrix with conducting components such as conductive polymers (CPs), metallic nanoparticles, or carbon materials [12]. Due to its tissue-like softness and the inherent presence of electrical fields similar to the innate nervous system, the conductive hydrogel can provide mechanical and electrical cues for enhancing neuronal differentiation of neural stem cells (NSCs) and controlling neurite extension $[11,13]$. We previously developed a porous, highly 
conductive, soft, and biocompatible conducting polymer hydrogel, which forms a free-standing conductive hydrogel for implantation into the spinal cord hemisection gap, and recently demonstrated that implanting this conductive hydrogel after SCI stimulated endogenous NSCs recruitment and neuronal differentiation after SCI [14]. Although conductive hydrogels effectively enhanced neuronal and axonal regeneration, their efficacy is compromised by host recognition and the subsequent foreign body immune responses, which cannot attenuate or even aggravate the early secondary inflammation after acute SCI [10, 12, 15]. Thus, single transplantation of conductive hydrogel may not be sufficient to achieve a substantial improvement in SCI repair.

Bone marrow stem cell (BMSC) therapy shows characteristic immunomodulatory effects and has been applied in severe clinical inflammatory diseases such as pancreatitis, colitis, and focal cerebral ischemia [16]. Exosomes (exos) are involved in intercellular communication and act on the innate immune system as paracrine messengers. They also exert immunomodulatory effects and can alleviate immune abnormalities $[17,18]$. Recently, BMSC-exos have emerged as a new cell-free therapeutic platform for various diseases due to their therapeutic effects, including their ability to promote regeneration and modulate immunoreaction [19]. In view of this, we hypothesized that a conductive hydrogel combined with BMSC-exos might achieve adequate therapeutic effectiveness in patients with SCI. Moreover, the delivery of BMSC-exos in conductive hydrogels can attenuate adverse host immune effects while synergistically exerting the therapeutic effect of promoting neurogenesis and axon growth, thereby alleviating SCI. Herein, we developed exos-loaded dual-network conductive hydrogels composed of photo-cross-linkable gelatin methacrylate (GM) hydrogels and polypyrrole (PPy) hydrogels. First, the GM/PPy (GMP) hydrogel scaffold was fabricated by in situ growth of the PPy network cross-linked and doped by tannic acid (TA) within the GM hydrogel networks, which possesses natural cell binding motifs such as Arg-Gly-Asp (RGD), allowing cells to grow within it. Then, BMSC-exos were immobilized in the TA-doped GMP hydrogel network to form a GM/PPy/Exos (GMPE) hydrogel via reversible hydrogen bond formation due to the presence of large amounts of polyphenol groups in TA. The noncovalent binding did not affect the structure and bioactivity of the exos while ensuring a slow sustained release of exos early in the implantation. Cell biocompatibility, adhesion, growth, and differentiation on the GMPE hydrogel scaffold were evaluated in vitro. In addition, the specific signaling pathways via which GMPE hydrogels manipulate immune response and promote axon regeneration were identified. A mouse spinal cord hemisection model was established to detect whether the GMPE hydrogel was efficient in facilitating nerve regeneration and improving functional recovery after SCI.

\section{Results}

\section{Identification of BMSCs and BMSC-exos}

The extraction of BMSCs and BMSC-exos is illustrated in Fig. S1A. Photomicrographs showed that primary BMSCs typically exhibited spindle-like morphology (Fig. S1B). Flow cytometry 
showed that the obtained cells expressed high levels of the positive BMSCs surface markers such as CD29, CD90, and CD44H, but did not express negative surface markers such as CD11b and chondrogenic differentiation (Fig. S1C), which also indicated the successful extraction of BMSCs. The presence of a cup-shaped morphology was observed with TEM analysis (Fig. S1D), a particle size of 70 to $140 \mathrm{~nm}$ from qNano measurement (Fig. S1E), and the expression of Flotillin-1, CD63, and TSG101 on the nanoparticles surface (Fig. S1F) all demonstrated successful extraction of BMSC-derived exos.

\section{Fabrication of GM, GMP and GMPE hydrogels}

We used a three-step synthesis process to produce GMPE hydrogels (Fig. 1B). Firstly, the GM hydrogel networks were formed by UV light photocrosslinking of GM units. Secondly, the GM hydrogel was successively immersed into solution I containing the monomers Py and TA and solution II containing APS, allowing in situ polymerization and crosslinking of conducting PPy chains. TA is an abundant natural water-soluble polyphenol present in plants that can act as a dopant and crosslinker for PPy hydrogel formation [14]. In this study, TA interacted with the amide bond on the GM backbone via hydrogen bonds and also reacted with PPy chains by protonating the itrogen groups on PPy to form a dual-network conductive hydrogel (GMP hydrogel) with strong interactions. This GM network conferred biocompatibility, tissue-like softness, degradability, and tissue and cell adhesion, while the PPy network provided conductive electrical activity to the hydrogels. Finally, the BMSC-exos were immobilized into the GMP hydrogel network to form the GMPE hydrogel via reversible hydrogen bond formation between the presence of a large amount of polyphenol groups in TA and the phosphate groups in the phospholipid of exos.

\section{Characteristics of GM, GMP, and GMPE hydrogel}

In comparison with gelatin, GM showed two new peaks at $5.3 \mathrm{ppm}$ and $5.5 \mathrm{ppm}$ that were attributed to the two protons of its methacrylate groups (Fig. S2A). The GM hydrogel exhibited amide bands characteristic of gelatin, including $\mathrm{C}=\mathrm{O}$ stretching at $1650 \mathrm{~cm}^{-1}$ (amide I), $\mathrm{C}-\mathrm{N}$ at $1440 \mathrm{~cm}^{-1}$, and $\mathrm{N}-\mathrm{H}$ deformation at $1239 \mathrm{~cm}^{-1}$ (amide III). In the PPy spectrum, the peaks at 1556 $\mathrm{cm}^{-1}$ and $1403 \mathrm{~cm}^{-1}$ are Py ring vibrations. These peaks also appeared in the spectra of the GMP hydrogels, indicating that the PPy chain was successfully incorporated into the GM hydrogel backbone (Fig. S2B). SEM analysis showed that GMPE hydrogels exhibited a three-dimensional highly porous structure (Fig. 1C), which provided space for nerve cell extension. In addition, high-magnification SEM also showed the interconnected globular nanoparticle morphology of PPy was coated onto the GM backbone (Fig. S2C). The mechanical properties of all samples were tested using dynamic oscillatory frequency sweep measurements. The storage moduli (elastic modulus, $G^{\prime}$ ) of all hydrogels were larger than the loss moduli (viscous modulus, $G^{\prime \prime}$ ) over an angular frequency range of 1-100 Hz, indicating that the hydrogels had good stability (Fig. S2D). The average storage moduli at a $10 \mathrm{~Hz}$ angular frequency increased from $555.7 \pm 50.1$ for the GM hydrogel to $1039.3 \pm 89.3 \mathrm{~Pa}$ and 1056.0 $\pm 133.1 \mathrm{~Pa}$ for the GMP and GMPE hydrogels, 
respectively (Fig. S2E). However, the mechanical properties of all three hydrogels matched neural tissue mechanics (600-3000 Pa), which was beneficial for cell function and differentiation. The introduction of hydrophobic PPy reduced the swelling ratio in the GMPE and GMP hydrogels when compared with GM hydrogel, although the difference was not statistically significant (Fig. S2F). Additionally, after soaking the hydrogel in the physiological medium for 7 and 14 days, the swelling ratio and mechanical properties of the GMPE hydrogel did not change significantly (Fig. S2G-H), indicating that the hydrogel exhibited long-term swelling effect and mechanical stability. Ex vivo spinal cords were able to stick to the GMPE hydrogel which indicated that the hydrogels also had excellent bioadhesion and supported their potential use in an in vivo animal SCI model (Fig. S2I).

To probe the electrochemical properties of GMP and GMPE hydrogels, a hydrogel electrode was prepared by in situ gelation of conductive hydrogels onto a piece of ITO. CV and EIS were performed with 0.1 M PBS (pH 7.4) as the electrolyte. Compared to the GM hydrogel, the GMP and GMPE hydrogels showed significantly improved anodic and cathodic currents (Fig. 1D). The CV curves showed similar oxidation and reduction current values for GMP and GMPE hydrogels. The EIS imaging showed a quasi-semicircle are appears in the high-frequency region of the Nyquist plots of the GMP and GMPE hydrogels, indicating that they exhibited good redox activity. Additionally, the diameter of this quasi-semicircle was related to the charge transfer resistance. The larger the radius of the circle, the larger the charge transfer resistance. The diameter of the semicircle for the GM hydrogel was significantly larger than that for the GMPE and GMP hydrogels, which indicated that the GMP and GMPE hydrogels both exhibited better electrical performance in comparison with GM hydrogels. In addition, the I-V curves showed that the conductivities of GMP and GMPE hydrogels were $1.83 \times 10^{-3}$ and $1.49 \times 10^{-3} \mathrm{~S} / \mathrm{cm}$, respectively, which were significantly higher than those of the GM hydrogel. As the Bode plots show, both GMP and GMPE hydrogels showed significantly low impedance values that were between $300 \mathrm{~Hz}$ and $1 \mathrm{kHz}$ compared with GM hydrogel. These values are within the frequency of the exchange signals observed in nerve cells [14,21]. Together, these data show that GMP and GMPE hydrogels exhibited similar electrical properties to each other, indicating that the introduction of exos had no obvious effect on the electrical properties of the conductive hydrogels. An isolated spinal cord circuit test was used to further evaluate the ability of the hydrogel to transmit electrical signals. After the ex vivo mouse spinal cord was transected, no electrical signal transmission was recorded below the injury site (Fig. 1E). When two ends of injury site were bridged by GMPE hydrogel, the stimulating electrical signals were able to be transmitted (Fig. $1 \mathrm{E})$, which indicated that the GPME hydrogel could partly restore endogenous electrical signaling transmission. In addition, the GMPE hydrogel also exhibited electrical stability in physiological medium for more than 2 weeks (Fig. S2J), which shows that it has potential property for longterm in vivo use.

The retention of BMSC-exos in GMPE hydrogel were investigate to evaluate the delivery capacity of the hydrogels. A three-dimensional IF image showed the uniform loading of PKH26 
labeled exos within the GMPE hydrogel (Fig. 1F). After the exos were immobilized in the hydrogel, the GMPE hydrogels were stored in PBS at $4^{\circ} \mathrm{C}$, and the retention of exos loaded in hydrogel was assessed over time with laser scanning confocal microscopy (Fig. S3A). These results revealed that exos retention times for the GMPE hydrogel were up to 14 days (Fig. S3B) and, with more than $90 \%$ of the exos exhibiting unobstructed release (Fig. S3C). The release of loaded exos from the GMPE hydrogel was also confirmed for up to 14 days, at which time exos could fully exert their immune-modulating and neurogenesis-enhancing effects. This observation was correlated to the strong affinity of TA in hydrogels with biomacromolecules. In vivo live imaging of PKH26 labeled exos showed they loaded within the hydrogel and remain at the injury site for 5 days after implantation, but the exos delivered in PBS were almost undetectable at the injury site (Fig. S3D). Moreover, the histological test further confirmed that the exos were phagocytosed by endogenous cells in vivo (Fig. S3E). These results indicated that hydrogels prolonged the residence time of exos and extended their release in the injury area.

To predict the possible mechanism of BMSC-exos on axon regeneration, neurogenesis and antiinflammatory function, the expression levels of related miRNAs in exos were measured. The GMPE BMSC-exos highly expressed axon regeneration-related miRNAs including miR29a, miR21, miR29c, miR26a, miR20a, miR9-5p, miR182, miR128, miR133b, miR431, and miR124; neurogenesis-related miRNAs, including miR145a, miR9-5p, miR148b, miR138, miR338, miR219a, miR1a; and anti-inflammatory-related miRNAs, including miR199a, miR99a, miR146a, miR181a, and miR411. Among them, miR29a, miR21, miR29c, miR26a, miR145a, and miR199a were relatively high in BMSC-exos (Fig. 1G). Moreover, PKH26 labeled exos were clearly detected in the cytoplasm of BV2 cells, suggesting successful in vitro endocytosis of exos released from the GMPE hydrogel (Fig. 1H). The level of the anti-inflammatory-related miRNAs in BV2 was significantly higher after endocytosis of BMSC-exos (Fig. 1I), especially, miR199a levels were nearly 3-fold higher in BV2 cells (Fig. 1I). NSCs can also normally phagocytize exos released from the GMPE hydrogel and the axon regeneration-related and neurogenesis-related miRNAs in NSCs significantly up-regulated after endocytosis of exos (Fig. 1J-K). In particular, miR21 was upregulated 4-fold and miR145a was upregulated 3-fold in NSCs (Fig. 1K).

\section{Biocompatibility and biodegradability of the hydrogels}

The viability of cultured cells was tested using live/dead staining and a CCK8 assay. The density of dead NSCs on the GMP hydrogel was significantly higher than that on the GM hydrogel (Fig. S4A). However, after exos were immobilized into the GMP hydrogel, the cell viability in the GMPE hydrogel cultures significantly increased, even more than that of the GM hydrogel group, as indicated by the live/dead staining and CCK8 assay results (Fig. S4A-B). Cytoskeleton staining was performed 3 days after cell were cultured in each condition (Fig. S4C). NSCs adhered to the plate surface and all three hydrogels. The spreading area and synaptic length of the cultured cells were significantly more on the GMPE hydrogel when compared to the other three groups. These results demonstrated that exos could function as biologically active microparticles that increased the in vitro cytocompatibility of the conductive hydrogels. 
Subcutaneous implantation of the hydrogels was performed in mice to test their in vivo degradation. Three weeks after implantation, the GM hydrogel almost completely degraded, while the GMPE and GMP hydrogels were still detected 6 weeks after implantation (Fig. S5A). These results indicated that the degradation rate of the GMPE and GMP hydrogels was significantly lower than that of the GM hydrogel. However, the volume of the GMP and GMPE hydrogels in both the diameter and thickness was reduced 6 weeks after implantation when compared to their pre-implantation volumes. In particular, the border of the conductive hydrogels became less defined (Fig. S5A, green arrow), suggesting that the conductive hydrogels degraded to a certain extent within 6 weeks. HE staining also showed that a small amount of mononuclear inflammatory cells invaded the GPE and GMPE hydrogels, which corresponded with the degraded interface between the tissue and the conductive hydrogels 6 weeks after hydrogel implantation (Fig. S5B). Additionally, histological staining revealed that the PPy chain broke into PPy nanoparticles and that the cytoplasm of monocytes contained a large number of PPy nanoparticles, which suggested that the endocytosis of PPy nanoparticles may contribute to PPy degradation.

On the other hand, the histological staining analysis of the hydrogel implantation site also revealed the formation of a fibrous capsule around the GMP and GMPE hydrogels 1 week after hydrogel implantation, indicating collagen deposition, which is a part of a normal inflammatory response (Fig. S5B-C). However, the fibrotic capsule of the GMPE hydrogel was significantly thinner than that of the GMP hydrogel (Fig. S5C). Moreover, the density of invasive mononuclear inflammatory cells significantly decreased 6 weeks after implantation, especially in the GMPE hydrogel group. These results demonstrated that exos decreased the inflammatory responses initially caused by the conductive hydrogels. In addition, the histological analysis confirmed that there was no obvious accumulation of hydrogel degradation products and no noticeable pathological abnormalities in the major organs (i.e. heart, liver, spleen, lung and kidney) in the mice treated with each hydrogel when compared with the control group (Fig. S5D). Moreover, there were no significant variations in the levels of alanine aminotransferase (ALT), aspartate aminotransferase (AST), and total protein (TP) in the GM, GMP, and GMPE hydrogel groups compared to the sham group, suggesting the hydrogels did not cause systemic toxicity (Fig. S5E). The in vitro hemolytic property is a standard method of assessing the hemocompatibility of biomaterials. Serum extracted from whole blood and hydrogel co-cultures had a clear yellow color similar to that of the PBS control group, but the Triton-100X group was bright red in color (Fig. S5F). The OD value of serum from three hydrogel groups was also similar to PBS group and that of all groups was significantly lower than that of the Triton-100X group (Fig. S5G). The hemolysis ratios of the three groups were all below 1\%, which indicated that the GMPE hydrogels had excellent hemocompatibility as nerve repair materials.

\section{GMPE hydrogel promotes M2 microglial polarization}

To assess whether the GMPE hydrogel indeed acted as an anti-inflammatory agent, we cocultured the GMPE hydrogel with a mouse microglia BV2 cell line. Microglia with specific 
markers can be divided into pro-inflammatory M1 or anti-inflammatory M2 phenotypes (Fig. 2A). The M1 markers mainly include the protein inducible nitric oxide synthase (iNOS), interleukin (IL)-6, and tumor necrosis factor alpha (TNF- $\alpha$ ), while M2 markers include arginase-1 (Arg-1) and IL-10 [22]. Gene expression showed that mRNA levels of the anti-inflammatory cytokines Arg-1 and IL10 were significantly higher in the GMPE group than in the GMP group. The mRNA expression levels of the pro-inflammatory cytokines iNOS, IL-6, and TNF- $\alpha$ in BV2 cells cultured on GMPE hydrogel were significantly lower than those in cells cultured on the GMP hydrogel (Fig. 2B). IF imaging of the cell cultures showed that the number of iNOS-positive cells was significantly higher, while the number of Arg-1-positive cells was significantly lower in the GMP hydrogel than in the control and GM groups (Fig. 2C-D). After exos were introduced into the conductive hydrogels, the number of iNOS-positive cells significantly decreased and the number of Arg-1-positive cells was significantly increased. Inflammation regulation, including iNOS and Arg-1 protein expression, was evaluated by WB analysis, which demonstrated that exos inhibited the inflammation caused by the conductive hydrogels (Fig. 2E, G). To investigate the possible mechanism by which the GMPE hydrogel repopulated microglia, we further assessed the relative levels of proteins in the inflammatory $\mathrm{NF}-\kappa \mathrm{B}$ pathway (Fig. $2 \mathrm{~F}, \mathrm{G}$ ). The GMPE hydrogel treatment inhibited the NF- $\mathrm{BB}$ pathway, indicated by the decreased expression of phosphorylated-

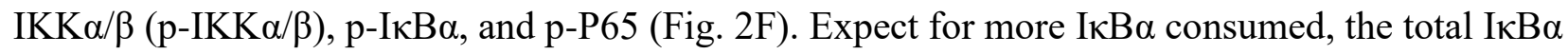
level in the GMP hydrogel was significantly lower than that in the GMPE hydrogel, while the total amounts of IKK $\alpha$, IKK $\beta$, P65 proteins were not reduced (Fig. 2F, G). The phosphorylation of I $\mathrm{B} \alpha \alpha$ protein was irreversibly inhibited by BAY 11-7082, and significantly reduced the level of $\mathrm{p}-\mathrm{I} \kappa \mathrm{B} \alpha$ (Fig. S6A). Additionally, p-P65 protein expression in BV2 cells cultured on conductive hydrogels was significantly lower than that in cells cultured without inhibitors (Fig. S6B-C). Furthermore, the level of Arg-1 protein was significantly higher, while that of iNOS protein was significantly lower in the BAY 11-7082 inhibition group compared to that in the samples that were not treated with BAY 11-7082. These results demonstrated that exos promoted microglia $\mathrm{M} 2$ polarization may through $\mathrm{NF}-\kappa \mathrm{B}$ pathway.

\section{NSCs differentiation on hydrogels}

NSCs are multipotent, self-renewing cells with the potential to differentiate into three cell sublineages, astrocytes, oligodendrocytes, and neurons (Fig. 3A) [23]. Compared to the control group, GMP and GMPE hydrogels promoted the expression of the neuronal differentiation marker $\beta 3$ tubulin (Tuj-1) and the oligodendrocyte differentiation marker myelin basic protein (MBP); however, the expression of the astrocyte marker glial fibrillary acidic protein (GFAP) gene was inhibited in these culture conditions (Fig. 3B). Furthermore, NSCs cultured on the GMPE hydrogel expressed the highest levels of MBP and the lowest levels of GFAP in comparison to the GMP hydrogel culture conditions, although the expression of GFAP showed no significant difference between the GMPE and GMP groups. The IF imageing and WB analysis showed (Fig. $3 \mathrm{C}-\mathrm{E}$ ), the protein expression for MBP and GFAP was consistent with their gene expression. After NSCs were cultured on GMP and GMPE hydrogels for 7 days, the levels of Tuj-1 and MBP 
protein both increased, while GFAP protein expression was decreased. Furthermore, when compared with the GMP group, the GMPE group showed significantly increased MBP expression. These results indicated that GMPE hydrogel promoted oligodendrocyte differentiation in NSCs, while inhibiting astrocyte differentiation.

\section{Axon outgrowth in differentiated NSCs grown on hydrogels}

The expression of the axon-associated proteins neurofilament (NF) and growth associated protein43 (GAP43) was used to investigate the effect of hydrogels on regenerative axon growth. The NF gene levels in the GMPE group were more than 30-fold higher than those in the control and GM groups and were more than 10-fold higher than those in the GMP group (Fig. 4B). Although the GAP43 level was higher in the GMPE groups than in the GMP group, the difference between the two groups was not significant. However, GAP43 expression levels in the GMPE group were significantly higher than those in the GM and control groups. Axon outgrowth from NF/GAP43positive cells and the formation of a new neural synaptic network was observed after 7 days in NSCs cultured on GMPE hydrogel (Fig. 4C). The axon density in the GMPE hydrogel group was the highest, with an axon length of $199.23 \pm 38.53 \mu \mathrm{m}$, which was relatively longer than that in the GMP $(118.82 \pm 15.76 \mu \mathrm{m}), \mathrm{GM}(79.64 \pm 12.14 \mu \mathrm{m})$ and control $(43.79 \pm 7.95 \mu \mathrm{m})$ groups (Fig. 4D). The protein levels of NF and the GAP43 postsynaptic marker were also increase in the GMPE group, which consistent with gene expression and protein localization data (Fig. 4E, F). Together, these results indicated that the GMPE hydrogel promoted axon outgrowth and neural synaptic network formation. To investigate the possible mechanism of axon extension, we measured the relative protein expression of the phosphate and tension homology deleted on chromosome ten (PTEN)/ phosphatidylinositol 3-kinase $(\mathrm{PI} 3 \mathrm{~K}) /$ protein kinase $\mathrm{B}(\mathrm{AKT}) /$ mammalian targets of the rapamycin (mTOR) pathway (Fig. 4E, F). A significant decrease in PTEN was observed in the GMPE group compared with the other three groups. Additionally, although the expression levels of phosphorylated (i.e. active) PI3K (p-PI3K), p-AKT, p-mTOR, and p-P70S6K were significantly upregulated in the GMPE group, the total amount of these proteins was not different between the tested growth conditions. The p-PI3K, p-AKT, p-mTOR, and p-P70S6K expression levels were also higher, while PTEN expression was lower in the GMP hydrogel than in the GM and control groups. These data validated our hypothesis that conductive hydrogels with exos promoted axon spread via the co-stimulation of the PTEN/PI3K/AKT/mTOR pathway. To further verify our hypothesis, p-mTOR expression was downregulated using m-TOR siRNA (Sim-TOR) and rapamycin (Rp) after NSCs cultured on the GMPE hydrogel. The siRNA directly binds the target mRNA, while Rp inhibits target protein phosphorylation, which both lead to a significant decrease in the p-mTOR protein levels (Fig. S7A). Rp was dissolved in dimethyl sulfoxide (DMSO); therefore, we included a DMSO group as a control and found that DMSO had no effect on the experimental outcome. The siRNA\#1 construct had the greatest effect on the target gene compared to the other clones; therefore, it was used for further experiments (Fig. S7B). When the PTEN/PI3K/AKT/mTOR pathway was downregulated, the protein expression levels of both NF and GAP43 decreased in the siRNA and Rp groups (Fig. S7C-D). Protein quantification 
and localization demonstrated that both axon density and length of the spread from the neuron differentiated from NSCs decreased when the pathway was inhibited (Fig. S7E-F). These findings further demonstrated that GMPE hydrogel promoted axon extension possible by activating PTEN/PI3K/AKT/mTOR pathway.

\section{The GMPE hydrogel improved mouse pathology and motor function after SCI}

The Basso mouse scale (BMS) score was used to evaluate mouse functional recovery after SCI. Each mouse exhibited normal locomotor activity of the right hindlimbs before injury ( 9 score). Immediately after right spinal cord hemisection, the animals exhibited complete paralysis ( 0 score) of the right hindlimbs (Fig. 5C). The right hindlimb BMS scores were no more than 2 in the SCI and GM groups 6 weeks after SCI, indicating a limited capacity for organism self-healing. In contrast, statistically significant locomotor functional recovery was observed in the GMPE groups in the 2 weeks post-operation, suggesting that the GMPE hydrogel promoted functional recovery at an early stage after SCI. In the GMP hydrogel group, the locomotor function was similar to that in the SCI and GM hydrogel groups 2 weeks post-injury, but significantly improved 4 weeks after SCI, although this improvement was significantly lower than that in the GMPE hydrogel. These findings showed that conductive hydrogels could partially promote mouse movement recovery. Six weeks post-operation, most GMPE hydrogel-implanted mice presented normal weight support (7 score), while most mice treated with the GMP hydrogel exhibited plantar placement with support (5 score) and mice in the SCI and GM groups exhibited extensive ankle movement (1-2 score). These results strongly suggest that the combination of conductive hydrogels and exos significantly improved functional recovery after SCI.

The footprint analysis results were consistent with the BMS scores and are summarized in Fig. 5D. Mice treated with the GMP hydrogel exhibited plantar placement with support but stepped without coordination; however, mice treated with the GMPE hydrogel exhibited coordinated crawling using their front and rear limbs, while mice still dragged their right hindlimbs in the SCI group. Six weeks after SCI, toe dragging and base of support were significantly reduced in the GMPE groups, suggesting these mice had restored weight bearing in comparison with those in the SCI, GM, and GMP groups (Fig. 5E). Furthermore, neither mean stride length nor the rotation angle improved in the GMPE group when compared with the other three groups, indicating hydrogel implantation improved coordination between the fore- and hind- paws. Footprint analysis further demonstrated that conductive hydrogels with exos enhanced coordination between the fore- and hind- paws.

Conventional MRI is routinely used for qualitative assessment of the injured spinal cord pathology (Fig. S8). Mice in the sham group showed normal spinal cord morphology, while those in the SCI and GM groups, the cross-sectional areas at the injury site of the spinal cord showed that the normal pathology of the spinal cord could not be seen 6 weeks after injury (Fig. S8). Similarly, right hemisection spinal cord defect normal morphology from the loss of normal nervous tissue at the injury site were observed in tissue HE staining (Fig. 5F). In transverse spinal cord sections, the cavitary area was $1.23 \pm 0.08 \mathrm{~mm}^{2}$ in SCI groups and was $1.08 \pm 0.05 \mathrm{~mm}^{2}$ in 
GM groups (Fig.5 G, J). In contrast, both the GMP and GPME hydrogels reduced vacuolation and the cavitary area in the GMPE group $\left(0.68 \pm 0.07 \mathrm{~mm}^{2}\right)$ was significantly smaller than that in the GMP group $\left(0.31 \pm 0.07 \mathrm{~mm}^{2}\right) 6$ weeks after surgery. The longitudinal cavitary area was $0.05 \pm$ $0.01 \mathrm{~mm}^{2}$ in GMPE group mice, which was significantly smaller than that of the SCI $(1.27 \pm 0.07$ $\left.\mathrm{mm}^{2}\right)$, GM $\left(0.99 \pm 0.02 \mathrm{~mm}^{2}\right)$ and GMP $\left(0.27 \pm 0.03 \mathrm{~mm}^{2}\right)$ groups (Fig. $\left.5 \mathrm{H}, \mathrm{I}, \mathrm{J}\right)$. These results confirmed that the GMPE hydrogel reduced the cavitary area and facilitating cell infiltration and tissue formation. Electrophysiological analyses were performed to estimate the degree of functional recovery. After the spinal cord above the injury site was provoked with a stimulating electrode, the activity of the target muscle was recorded by a receiving electrode, reflecting the functional recovery after SCI (Fig. 5K). The change in CMAP signal amplitude was recorded from the gastrocnemius and analyzed individually. CMAP signals from the mouse right hindlimbs in the SCI group decreased almost 5-fold in comparison to those in the sham group 6 weeks postSCI (Fig. 5L). In contrast, the CMAP signal amplitude recorded from the GMPE group was 1.82 $\pm 0.06 \mathrm{mV}$, which was significantly greater than that in the GM $(0.59 \pm 0.19 \mathrm{mV})$ and SCI $(0.45 \pm$ $0.10 \mathrm{mV}$ ) groups (Fig. 5M). Although the amplitude $(1.36 \pm 0.11 \mathrm{mV})$ in the GMP hydrogel group was significantly lower than that in the GMPE hydrogel, it was still greater than that in the SCI and GM groups. These data demonstrated that the GMPE hydrogel was superior to the GMP hydrogel in improving motor function after SCI.

\section{Hydrogels modulate inflammation in vivo}

Inflammatory reactions usually lead to a series of secondary insults after SCI. Therefore, inhibition of inflammation can provide a good environment for SCI repair. Seven days after SCI, the spinal cords of mice in each treatment group were harvested to evaluate the inflammation caused by the injury and materials. The density of CD68-positive cells was significantly greater at the injury site in the SCI group than in the sham group, indicating that severe inflammation occurred early after SCI (Fig. S9A). The CD68 IF intensity increased in the GMP group at the center of the injury site and was higher than that in the SCI group (Fig. S9B), which suggested that conductive hydrogels further aggravated local inflammation. Transplantation of the GMPE hydrogel significantly reduced the density of CD68-positive cells and the expression levels of pro-inflammatory factors (iNOS), while increasing the expression levels of anti-inflammatory factors (Arg-1). These results demonstrated the immunomodulatory properties of BMSC-exos (Fig. S9C-D). This result was similar to that observed in subcutaneous implantations as previously reported and demonstrated that implantation of the GMPE hydrogel could inhibit the inflammatory response at an early stage of SCI.

\section{Hydrogels enhanced neurogenesis in vivo}

To further investigate the histological changes and the mechanism underlying functional recovery of SCI after GMPE hydrogel treatment, protein expression and localization was used to access local neurogenesis and astrological scar formation at the injury site. Neurogenesis included the proliferation, migration, and neural differentiation of the endogenous NSCs. Six weeks after injury, numerous nestin-positive NSCs appeared around the injury site in the GMP and GMPE 
groups (Fig. 6A-B), as well as migration of nestin-positive NSCs into the injury site in the GMP and GMPE hydrogel groups. However, nestin-positive NSCs in the SCI and GM hydrogels were almost undetectable at the lesion center. In the GMP and GMPE hydrogel groups, we also observed large numbers of Tuj-1-positive, newborn neurons at the center of the lesion area, while these cells were relatively less present in the GM hydrogel, and SCI groups (Fig. 6C-D). However, the intensity of Tuj-1 positive neurons was not significantly different among the SCI, GM, GMP, and GMPE groups at the rostral and caudal borders. These findings suggest that conductive hydrogels promoted the invasion of newborn neurons into the hydrogels and promoted neurogenesis. Moreover, the number of GFAP-positive astrocytes significantly increased at the caudal and rostral regions and formed an astrological scar around the lesion site in GM and SCI groups, which was more hypertrophic than that in the GMP and GMPE hydrogel groups (Fig. 6C, E). Thus, GMP and GMPE hydrogels inhibited GFAP-positive astrocytic scar formation, providing a favorable microenvironment for later axon regeneration. WB analysis was consistent with the IF results; the expression of Tuj-1 in the GMPE group was similar to that in the GMP hydrogel but was much higher than that in the SCI and GM groups, while GFAP at the lesion site in the GMPE group was also similar to that in the GMP group and significantly lower than that in the GM and SCI groups (Fig. 6F-G). Together, these results show that the GMPE hydrogel enhanced neuronal differentiation and inhibited astrocytic differentiation.

\section{Axon regeneration and remyelination in vivo}

Axon regeneration was quantified by determining the NF-positive axons that regenerated into the lesion site. Longitudinally oriented NF-positive fibers that originated from newly regenerated neuronal cells were observed in both the GMP and GMPE groups (Fig. 7A). However, these axons were wrapped around the material and appeared disordered in the GMP hydrogel group, (Fig. 7A, white arrows). The NF positive axons formed tended to connect both ends of the injury site in the GMPE group, while the axon density between the GMP and GMPE hydrogel groups was similar at the rostral/caudal borders. However, when we compared axon density at the injury site, it was highest in the GMPE group, relatively low in the GMP hydrogel treatment, and much lower in the SCI and GM groups (Fig. 7B). These results demonstrate that GMPE hydrogel transplantation can induce axonal regeneration at the injury site. Furthermore, the regenerated axons in both the GMP and GMPE groups showed typical wrapping with myelin sheets (Fig. 7A) and the MBP-positive myelin sheath density was highest in the GMPE hydrogel group at the center of the injury site when compared to the SCI, GM and GMP groups (Fig. 7B). In striking contrast, nearly no myelin-associated axons were detected at the caudal ends or at the lesion site center of the SCI, and fewer disordered myelin-associated axons were detected in the GM groups. Additionally, LFB staining showed that nerve myelin was detected around the GMPE hydrogel, while the density and the positive area of myelin were significantly lower in the other three groups (Fig. 7C). These results strongly suggest that the GMPE hydrogel significantly improved myelinated nerve fibers. WB analysis was consistent with the protein localization results, indicating that NF and GAP43 were expressed more at the injury site in the GMPE group that in 
the GMP, GM, and SCI groups (Fig. 7D-E). Consistent with our in vitro experiments, the relative phosphorylation of PTEN/PI3K/AKT/mTOR pathway proteins was highest in the GMPE group, and the protein expression levels in the GMP group were significantly upregulated when compared to those in the SCI and GM groups. These results further demonstrated that these axonal regeneration phenomena were associated with PTEN/PI3K/AKT/mTOR pathway activation (Fig. 7D-E).

\section{Discussion}

Neuroinflammation, glial scar formation, and difficulty in axon regeneration are the factors limiting regeneration capability after SCI [24]. Scaffold biomaterial-based treatment has been proposed as an appropriate axon guidance approach to promote neural tissue regeneration [7, 14]. The capacity to conduct electrical signals is an important requirement for the biomaterials used in neuroregeneration [13]. Our conductive hydrogels exhibited suitable swelling and mechanical properties, and excellent electrical conductivity that were similar to native neural tissue, making them promising candidates for nerve injury repair [10, 13]. The swelling ratio of the GMP and GMPE hydrogels was slightly reduced after PPy chain polymerized into the GM hydrogel, but still higher than the swelling ratio of the other reported conductive hydrogels [25]. A proper swelling ratio could facilitate efficient substance transfer and is beneficial for soft native nerve tissue engineering applications. As previously reported, the proliferation and differentiation of NSCs can be modulated by the mechanical properties of the materials [7]. In this study, the storage moduli of the GMPE hydrogel was 1056.0 $\pm 133.1 \mathrm{~Pa}$, which matched neural tissue mechanics (600-3000 Pa) and indicates its suitability for soft nerve tissues engineering. The conductivity of the GMP $\left(1.83 \times 10^{-3} \mathrm{~S} / \mathrm{cm}\right)$ and GMPE $\left(1.49 \times 10^{-3} \mathrm{~S} / \mathrm{cm}\right)$ hydrogels were both superior to that of the GM hydrogel and exceeded the reported conductivity of typical conductive hydrogels $\left(10^{-6}-10^{-5} \mathrm{~S} / \mathrm{cm}\right)$ [26]. Moreover, our GMPE hydrogel also showed good swelling, mechanical, and electrical stability for 14 days in physiological medium, which could facilitate their long-term use for nerve repair. Both GMP and GMPE hydrogels had low impedance. The high conductivity ability and low resistance of GMPE and GMP hydrogels are important for nerve cell intercellular signal transmission [14]. Electrical signals directly stimulate voltage-gated $\mathrm{Ca}^{2+}$ channels to increase the intracellular $\mathrm{Ca}^{2+}$ level that activates PTEN/PI3K/AKT/mTOR pathways, in which AKT plays important roles in regulating neuronal differentiation and axon regeneration [27]. Our in vitro results demonstrated that the GMP hydrogel significantly increased neuron and oligodendrocyte differentiation but reduced astrocyte differentiation in comparison with control and GM groups. Six weeks after the conductive hydrogels were implanted into the spinal cord hemisection gap, the GMP and GMPE hydrogels induced migration of nestin-positive endogenous NSCs into the injury site. Both hydrogels were surrounded by newborn neurons that even invaded into the hydrogels. Conversely, the SCI and GM groups formed an astrocyte scar surrounding the lesion site and no obvious newborn neurons were observed. Lesion site neurogenesis subsequently promotes neuron maturation and lead to the formation of a nascent 
functional synaptic network [28]. Oligodendrocyte differentiation and remyelination is another

important process for nerve function recovery. However, spontaneous remyelination often fails after SCI, primarily due to failure of oligodendrocyte differentiation rather than oligodendrocyte depletion [29, 30]. Hence, conductive hydrogels significantly improve oligodendrocyte differentiation that is critical for the myelin formation, which is essential for rapid and efficient action potential propagation and functional recovery [29, 31]. Our results showed plenty of myelin-associated axonal regeneration was guided by the GMP hydrogel into the lesion site. In comparison with the GM and control groups, the GMP group showed downregulation of PTEN levels and upregulation of p-PI3K, p-AKT, and their downstream signaling pathway, indicating that the conductive hydrogels modulated NSC differentiation and axon regeneration possibly through the PTEN/PI3K/AKT/mTOR pathway.

The inflammatory responses induced by SCI limits the therapeutic efficiency of biomaterials [1]. In this study, we found that conductive hydrogels significantly aggravated the inflammation after acute SCI by increasing the proportion of M1 microglia and increased the number of CD68positive microglia in comparison with that in the SCI group 7 days post-surgery. Microglia mediated-inflammation contributes to adverse host immune effects and can lead to the rejection of transplanted biomaterials used for SCI repair [32]. Recent studies suggest that the inflammation microenvironment of the injured spinal cord contributes to the transformation of antiinflammatory M2 microglia into pro-inflammatory M1 microglia shortly after injury and can last for weeks or months, which can act as a barrier to neural regeneration [33, 34]. Therefore, decreasing early-stage inflammation and regulating M1/M2 microglia polarization is critical for the application of engineered materials used in SCI repair. Previous studies have reported that BMSC therapies have immunomodulatory effects in severe clinical inflammatory diseases such as pancreatitis, colitis, and focal cerebral ischemia [35]. BMSC-exos are important paracrine soluble factors that act as main regulators of intercellular communication [17]. Exos range in size from 10 $-100 \mathrm{~nm}$ and contain proteins, mRNA, and miRNA molecules that negatively regulate relative gene expression on the post-transcriptional level [11].

In this study, we combined a conductive hydrogel scaffold with BMSC-exos for SCI therapy. The driving force for the interaction between TA and BMSC-exos the reversible non-covalent hydrogen bonds formed between the phosphate group in the phospholipids and polyphenol groups in TA that allowed the exos in the GMPE hydrogel to be sustainably released and detected for up to 14 days in vitro. Furthermore, NSCs and BV2 cells cultured on the GMPE hydrogel could normally phagocytize exos released from the hydrogel, indicating that exos still showed good activity. Meanwhile, in vivo GMPE hydrogel implantation showed local site exos retention. Exos expressed high levels of anti-inflammatory-related miRNAs, including miR199a, miR99a, miR146a, miR181a and miR411, with the miR199a level being the highest. After BV2 took in the exos released from the GMPE hydrogel, the levels of all miRNAs increased, especially miR-199a, which showed a nearly three-fold increase in its levels. Previous reports showed that miR-199a acts as a negative regulator of IKK $\beta$ and activates the IKK- $\beta-N F-\kappa B$ signaling pathway, which is 
the master regulator of innate immunity $[36,37]$. We found that the GMPE hydrogel promoted microglial polarization in vitro and that the expression levels of $\mathrm{p}-\mathrm{IKK} \alpha / \beta, \mathrm{p}-\mathrm{I} \kappa \mathrm{B} \alpha$, and $\mathrm{p}-\mathrm{P} 65$ significantly decreased in BV2 cells grown on the GMPE hydrogel when compared with the GMP hydrogel. In addition, BAY 11-7082 was used to inhibit phosphorylation of ІкB $\alpha$, which resulted in the downregulation of iNOS and upregulation of Arg-1 in BV2 cells cultured on the GMP hydrogel. Therefore, we suggest that the GMPE hydrogel modulated microglia mediatedinflammation, possibly through miRNAs carried by exos that suppressed NF-kB pathway activation. Furthermore, the in vivo results also demonstrated that GMPE hydrogel promoted fewer CD68-positive microglia than the GMP, GM, and SCI treatments at the early stages of injury. Alarming, we also found that the GMP hydrogel promoted CD68-positive microglia to localize to and further induce early inflammation at the injury site. The expression of iNOS was highest and Arg-1 was lowest in the GMP group compared to the other treatment conditions, which indicated that the GMP hydrogel could promote microglia M1 polarization in vivo. These findings indicate that the combined hydrogel-exos treatment largely resolved microglia mediatedinflammation at the injury site by reducing CD68 protein levels and pro-inflammatory factor release.

In addition, the delivery of BMSC-exos in conductive hydrogels can exert synergistic therapeutic effect on SCI. BMSC-exos also showed high expression levels of neurogenesis-related miRNAs (miR145a, miR9-5p, miR148b, miR138, miR338, miR219a, and miR1a) and axon regenerationrelated miRNAs (miR29a, miR21, miR29c, miR26a, miR20a, miR9-5p, miR182, miR128, miR133b, miR431, and miR124). Similar to the GMP hydrogel, the GMPE hydrogel also promoted NSC neuron and oligodendrocyte differentiation but inhibited astrocyte differentiation. Interestingly, the GMPE group showed significantly greater oligodendrocyte differentiation than the GMP group in both in vitro and in vivo studies. Two neurogenesis-related miRNAs (miR145a and miR148b) are primarily related to oligodendrocyte maturation, and miR145 is a critical regulator of the human analog of myelin regulatory factor, which is mainly responsible for oligodendrocyte differentiation and maturation [36]. The GMPE hydrogel was more efficient in promoting NF-positive axon regeneration due to the addition of BMSC-exos that highly expressed axon regeneration-related miRNAs. MiR29a, miR21, miR29c, and miR26a also promote axon growth by suppressing PTEN and activating the PI3K/AKT pathway [36]. Coincidentally, miR29a, miR21, miR29c, and miR26a were highly expressed in BMSC-exos and their levels significantly increased after NSC phagocytized exos, especially miR21, which was 4fold up-regulated. Meanwhile, after mTOR was selectively inhibited by siRNA and Rp, the length of the axon significantly decreased in GMPE hydrogels. Therefore, BMSC-exos combined conductive hydrogels promoted SCI repair mainly through increased NSC oligodendrocyte differentiation and myelin-associated axonal regeneration. Furthermore, we speculated that the GMPE hydrogel promoted myelin-associated axon regeneration by conductive hydrogels and exos co-activating the PTEN/PI3K/AKT/mTOR pathway. 
Improved myelin-associated axonal regeneration and reduced cavitary areas may explain how neural function repaired for locomotion was recovered by GMPE hydrogel treatment. GMP can partly promote myelin-associated axonal regeneration. Four weeks after GMP hydrogel treatment, the BMS score of the right hindlimb was significantly higher than that of the GM and SCI groups. However, it was still significantly lower than that of the GMPE hydrogel, which may be due to the synergistic effects of the embedded exos on axon regeneration. Six weeks after SCI, the right hindlimb behavior in the SCI and GM groups exhibited extensive ankle movement, while mice treated with the GMP hydrogel presented only limited weight bearing. In contrast, mice in the GMPE group presented weight bearing ability and partially restored coordination 6 weeks after spinal cord hemisection. Similarly, the amplitude of CMAP signal recorded from the gastrocnemius of the GMP group mice was significantly higher than that of the GM and SCI groups, while it was still significantly lower than that of the GMPE group. In addition, due to the early anti-inflammatory effects of GMPE hydrogel, mice treated with GMPE hydrogel showed ankle movement just 2 week post-SCI and their BMS scores were significantly higher than those of the SCI, GM, and GMP groups at same timepoint, further indicating that the GMPE hydrogel can promote faster and better functional recovery after SCI.

Our GMPE hydrogel also had good biocompatibility and suitable biodegradability. The conducting components in current conductive hydrogels, such as CPs, metal nanoparticles (NPs), or carbon-based materials, have very low biodegradability and poor biocompatibility [27, 38]. A non-degradable composition would require secondary surgery to remove the material from the injury site, which may inhibit tissue regeneration at the lesion site [38]. In contrast, degradable conductive hydrogels that match the tissue regeneration process can create a regenerationpromoting microenvironment and guide the replacement of the biomaterials with regenerating tissue. In this study, the GM hydrogels completely degraded in 6 weeks after implantation, suggesting enzymatic cleavage of the GM backbone [39]. Our dual-network GMP hydrogel was synthesized by growing PPy chain doped with TA onto the surface of a porous GM backbone with PPy component is constructed with interconnected nanoparticles. HE staining demonstrated that monocytes around the GMP and GMPE hydrogel could phagocytose PPy and accelerate PPy chain degeneration. Hence, we believe that the degeneration of our conductive hydrogels was likely a result of both enzymatic hydrolysis of the GM backbone and subsequent breakdown of the PPy chain into PPy nanoparticles. Previous study demonstrated that PPy nanoparticles biosafety in vivo and that they are taken up by mouse macrophage RAW 264.7 cells [14, 40]. Our histological staining also showed that the GMPE and GMP hydrogels were progressively biodegraded at the SCI lesion site, and that newborn neurons penetrated through the hydrogels to gradually replace them. Although the subcutaneous macroscopic view of the hydrogels implanted site showed that GMPE and GMP hydrogels were still present 6 weeks after subcutaneous implantation, the volume of these hydrogels was significantly reduced compared to that of initial implantation. Thus, nerve regeneration, which usually requires several months or years, could benefit from the slow degradability of conductive hydrogel implants. Additionally, degradation 
products of all three hydrogels showed no toxicity to the main internal organs (heart, liver, spleen, lung, and kidney) in the mouse model. Furthermore, the levels of ALT, AST, and TP in the mice treated with the three different hydrogels were similar to those in healthy mice. In vitro and in vivo data demonstrated that the long-term biocompatibility and suitable biodegradability of exosloaded conductive hydrogels are suitable for SCI repair. In future studies, a long-term in vivo study will be applied to assess the complete degradation process of our hydrogel implant. In summary, we have demonstrated that by immobilizing the BMSC-exos in the conductive hydrogels prepared in our study, inflammation inhibition, neurogenesis enhancement, and myelinassociated axonal regeneration promotion can be achieved for SCI therapy. With the reversible non-covalent binding, the BMSC-exos carried on the GMPE hydrogel showed good activity and could be sustainably released, allowing their accumulation in the mice spinal cord lesion site. GMP hydrogel implantation can promote NSC neurogenesis and axonal growth, but it inhibits scar-forming gliosis. However, single treatments with conductive hydrogels even aggravated inflammation at the injury site early after SCI, which reduced the efficacy and increased the adverse effects. Due to the immunomodulatory properties of BMSC-exos, the GMPE hydrogel regulated M1/M2 polarization from an M1- to M2-dominant phenotype via the NF- $\mathrm{kB}$ pathway. Moreover, the GMPE hydrogel could reduce CD68-positive microglia at an early stage after SCI, attenuating the adverse immune effects. In comparison with treatments using the GMP hydrogel alone, the GMPE hydrogel, a combination of exos and conductive hydrogels, further enhanced oligodendrocyte differentiation and myelin-associated axonal regeneration. Furthermore, in vitro and in vivo studies supported our findings showing that the GMPE hydrogel enhanced neuronal and oligodendrocyte differentiation of NSCs and promoted axon growth via exos and conductive hydrogels co-activating PTEN/PI3K/AKT/mTOR pathway. Thus, the GMPE hydrogel targeted three areas, namely, reduction of early inflammation, enhancement of neurogenesis, and promotion of myelin-associated axonal regrowth, which synergistically promoted locomotor recovery after mice spinal cord hemisection. This study suggests that a combination of conductive hydrogels and BMSC- exos would be a promising therapeutic strategy for SCI treatment.

\section{Materials and Methods}

\section{Isolation and characterization of mouse bone marrow mesenchymal stem cells (BMSCs)}

Bilateral humeri and femurs of C57BL/6J mice ( $\mathrm{n}=5$, 4-week-old) were extracted under sterile conditions to resect metaphyses of each bone. Low glucose Dulbecco's Modified Eagle Medium (DMEM, Gibco) containing 10\% fetal bovine serum (FBS, Gibco) and 1× penicillin/streptomycin was used to rinse the bones until the color of bone marrow tuned white. The mixture was then incubated at $37^{\circ} \mathrm{C}$ with $5 \% \mathrm{CO}_{2}$. The culture medium was renewed every three days to remove non-adherent cells. Surface marker antibodies were used as described previously to verify cells expressing CD11b, CD45 CD29, CD90, and CD44 (eBioscience). The differentiation of adipocytes, osteoblasts, and chondrocytes of extracted BMSCs was included as described previously [20]. Briefly, BMSCs were cultured in (1) osteogenic differentiation medium: DMEM 
containing 10\% FBS, $50 \mu \mathrm{g} / \mathrm{mL}$ ascorbic acid, $10 \mathrm{nmol} / \mathrm{L}$ dexamethasone and $10 \mathrm{mmol} / \mathrm{L} \beta$ glycerophosphate; (2) adipogenic differentiation medium: DMEM containing 10\% FBS, 50 $\mu \mathrm{g} / \mathrm{mL}$ ascorbic acid, $10 \mu \mathrm{g} / \mathrm{mL}$ insulin, $10 \mathrm{nmol} / \mathrm{L}$ dexamethasone, and $50 \mu \mathrm{g} / \mathrm{mL}$ indomethacin; or (3) chondrogenic differentiation medium: DMEM containing 1\% FBS, $50 \mu \mathrm{g} / \mathrm{mL}$ ascorbic acid, $100 \mathrm{nmol} / \mathrm{L}$ dexamethasone, $10 \mathrm{ng} / \mathrm{mL}$ transforming growth factor alpha (TGF- $\beta$ ), $6.25 \mu \mathrm{g} / \mathrm{mL}$ insulin, $6.25 \mu \mathrm{g} / \mathrm{mL}$ transferrin, $6.25 \mu \mathrm{g} / \mathrm{mL}$ selenous acid. The media were changed every 3 days. Osteoblasts, adipocytes, and chondrocytes were identified using Alizarin Red staining, Oil Red O staining, and Alcian Blue staining respectively, 14 days after culture.

\section{Isolation and characterization of BMSC-exos}

All BMSC-exos used in the in vitro and in vivo experiments were obtained from the same batch of BMSCs. FBS was ultracentrifuged at $120,000 \times \mathrm{g}$ under $4{ }^{\circ} \mathrm{C}$ for $12 \mathrm{~h}$ using an ultracentrifuge (Beckman Coulter) to prepare exos-free FBS. Exos-free culture medium was used to replace conventional culture medium as the cell density reached $80 \%$. After the culture medium was gradient centrifuged to remove dead cells and cell debris, the supernatant was centrifuged at $100,000 \times \mathrm{g}$ for $90 \mathrm{~min}$, and the isolated exos were resuspended in $50 \mu \mathrm{L}$ phosphate buffered saline (PBS, Gibco) for further use. The qNano ${ }^{\circledR}$ system (Izon Science) was used to detecte the size of exos, and transmission electron microscopy (TEM, HT7700, HITACHI) was used to analyze the morphology of exos. Western blotting (WB) was used to determine the expression of Flotillin-1 (Abcam), TSG101 (Abcam), and CD63 (ProteinTech). A BCA protein assay kit (Thermo Fisher) was used to detect the concentration of exos.

\section{MicroRNA gene expression}

The miRNeasy Mini Kit (Qiagen) was used to extract microRNAs (miRNAs) from exos. The primer information for each miRNA is provided in Table S1. U6 was set as the normalized miRNA expression. E. coli Poly(A) Polymerase (NEB) was used to catalyze the templateindependent addition of AMP from ATP to the $3^{\prime}$ end of miRNA. Reverse transcription for cDNA synthesis was performed using the PrimeScript ${ }^{\mathrm{TM}} \mathrm{RT}$ reagent Kit (Takara). Reverse transcription quantitative polymerase chain reaction (RT-qPCR) was performed using LightCycler 480 SYBR Green I Master (Roche).

\section{Synthesis of GM, GMP and GMPE hydrogel}

GM monomer was synthesized as previously described [7]. Briefly, $1 \mathrm{~g}$ gelatin was dissolved in $10 \mathrm{~mL}$ PBS (pH 7.4) at $50{ }^{\circ} \mathrm{C}$ to obtain $10 \%(\mathrm{w} / \mathrm{v})$ gelatin. Then, $0.5 \mathrm{~mL}$ methylacrylic anhydride (MA) was added into the gelatin solution and incubated for $1 \mathrm{~h}$ with stirring. Another $10 \mathrm{~mL}$ PBS was introduced to stop the reaction. The solution was dialyzed using deionized water and lyophilized for future use. The GM hydrogels were prepared by ultraviolet (UV) cross-linking of the GM monomer (3\% w/v) and 0.5\% photoinitiator (Irgacure 2959; Sigma). Solution I was prepared by dissolving $0.03 \mathrm{~g}$ TA and $70 \mathrm{uL}$ pyrrole (Py) in $10 \mathrm{~mL}$ deionized water. Solution II was prepared by dissolving $0.228 \mathrm{~g}$ ammonium persulfate (APS, Sigma) in $10 \mathrm{~mL}$ deionized water. The two solutions were then stored at $4{ }^{\circ} \mathrm{C}$ for $30 \mathrm{~min}$ before use. The GM hydrogels were immersed in solution I for $12 \mathrm{~h}$ and then solution II was added and the mixtures was incubated at 
$4^{\circ} \mathrm{C}$ overnight to synthesize the GMP hydrogel. GMP hydrogels were then incubated in DMEM basic overnight at $4{ }^{\circ} \mathrm{C}$ to clear any uncrosslinked side products. Finally, $200 \mu \mathrm{g}$ of BMSC-exos

\section{Electrical characterization}

$$
\text { Swelling ratio }(\%)=\frac{\mathrm{W} 1-\mathrm{W} 2}{\mathrm{~W} 2} \times 100
$$

Electrical characterization including cyclic voltammetry (CV), electrochemical impedance (EIS), and current-voltage (I-V) of each hydrogel was performed using an electrochemical workstation (Zennium Zahner) [14]. The working electrode was a hydrogel-coated Indium-Tin Oxide (ITO) glass. The counter and reference electrodes were a platinum mesh and $\mathrm{Ag} / \mathrm{AgCl}$, respectively. The $\mathrm{CV}$ measurements were performed at a scan rate of $10 \mathrm{mVs}^{-1}$, ranging from -0.5 to $1.0 \mathrm{~V}$ in 0.1 
M PBS. The EIS spectra were measured at open circuit potentials ranging from $100 \mathrm{kHz}$ to 0.01 Hz. The I-V was tested using a two-probe Keithley 2400 Sourcemeter in the range from -0.5 to $0.5 \mathrm{~V}$.

\section{Neural stem cell (NSC) isolation and BV-2 microglial cell (BV2 cell) culture}

Brains from E14 mouse embryos were dissociated into single cells with mechanical shearing and transferred into low-attachment dishes. Cells were cultured in DMEM/F12 (Gibco) supplemented medium containing with $1 \times$ B27 neuronal supplement (Gibco), $20 \mathrm{ng} / \mathrm{mL}$ epidermal growth factor (EGF; PeproTech), 20 ng/mL basic fibroblast growth factor (bFGF; PeproTech), 1× Glutamax (Gibco), and $1 \times$ penicillin/streptomycin (Gibco). The medium was changed every 3 days. The BV2 cells were purchased from the ATCC cell bank and cultured in high glucose DMEM medium containing $10 \%$ FBS, which was replaced every other day.

\section{Exos labeling and cell phagocytosis studies}

The PKH26 red fluorescent membrane linker dye (Sigma) was used to label BMSC-exos. Exos were resuspended with 500uL Diluent $\mathrm{C}$ solution, and then $5 \mathrm{uL}$ PKH26 red fluorescent dye was added to the suspension and incubated at $37^{\circ} \mathrm{C}$ for $5 \mathrm{~min}$. To neutralized the residual dye, $10 \mathrm{~mL}$ DMEM was added to the samples. Samples were ultracentrifugated at $100,000 \times \mathrm{g}$ for $1 \mathrm{~h}$ at $4{ }^{\circ} \mathrm{C}$ before the exos were combined with hydrogel for cell culture and implantation. To further detect exos phagocytosis, cytoskeleton staining was performed using Actin-Tracker Green (Beyotime). Cells were fixed with $4 \%$ paraformaldehyde for $30 \mathrm{~min}$, and then incubated in a membrane breaking solution containing $0.2 \%$ Triton-100X (Biofroxx) and $6 \%$ bovine serum albumin (BSA, Biofroxx) at $37{ }^{\circ} \mathrm{C}$ for $1 \mathrm{~h}$. Actin-Tracker Green was added, and then samples were incubated at $4{ }^{\circ} \mathrm{C}$ for another $1 \mathrm{~h}$. Hoechst 33342 (Sigma) stain was added for $5 \mathrm{~min}$ before samples were observed using a confocal reflection microscope (Leica).

\section{NSC viability, proliferation, and adhesion on the hydrogels}

The viability of NSCs was calculated using Calcein-AM/ Propidium iodide (Calcein-AM/PI, Invitrogen) staining $24 \mathrm{~h}$ after co-culture with each hydrogel as previously described [7]. CalceinAM and PI were added to double-stain NSCs for $15 \mathrm{~min}$ at $37{ }^{\circ} \mathrm{C}$ in $5 \% \mathrm{CO}_{2}$. The micrographs of living/dead cells were captured using a confocal reflection microscope (Leica). In addition, the Cell Counting Kit-8 (CCK-8, Dojindo) solution was added to 12-well NSCs culture plates at a ratio of 1:10 to detect cell proliferation on days 1,3 , and 7 . After incubation for $4 \mathrm{~h}, 100 \mu \mathrm{L}$ of supernatant mixed solution was transferred into 96-well plates and measured with an enzymelabeling instrument (SpectraMax M5) at the 450-nm wavelength. The cytoskeleton was stained with Actin-Tracker Green after 3 days of culture to observe cell adhesion and spreading.

\section{Gene expression}

A total RNA kit (Omega) was used to harvest mRNA from cells cultured on each hydrogel. Similarly, cDNA reverse transcription was performed using the PrimeScript TM RT reagent kit. RT-qPCR was performed using LightCycler 480 SYBR Green I master mix. The primers used in these experiments are listed in Table S1.

\section{Immunofluorescence (IF)}


Cells or tissues were fixed in $4 \%$ paraformaldehyde for $30 \mathrm{~min}$. Subsequently, samples were incubated in PBS containing $0.2 \%$ Triton-100X and $6 \% \mathrm{BSA}$ for $1 \mathrm{~h}$ at $37{ }^{\circ} \mathrm{C}$. The corresponding were added to the cells or tissues and incubated at $37{ }^{\circ} \mathrm{C}$ for 1 hour. Hoechst 33342 was used to stain nuclei before imaging. PBS was used to wash the samples three times between each step. Micrographs were captured using a confocal reflection microscope (Leica).

\section{WB assay}

Cells or tissues were lysed in RIPA lysis buffer (CWBIO) containing protease inhibitor (Thermo Fisher) and phosphatase inhibitor (Thermo Fisher). Protein concentration was determined using a BCA protein assay kit (Thermo Fisher). Equal amounts $(40 \mu \mathrm{g})$ of each protein suspension were separated on an $8 \%$ SDS-PAGE gel (Thermo Fisher) and then transferred onto polyvinylidene fluoride (PVDF, MILLIPORE) membranes. Membranes were subsequently blocked with 5\% skim milk for $1 \mathrm{~h}$ before being incubated with primary antibodies at $4{ }^{\circ} \mathrm{C}$ overnight. A specific secondary antibody (CST) was added to the membranes for $1 \mathrm{~h}$ before the immunoblots were visualized using an enhanced chemiluminescence (ECL) kit (Thermo Fisher). Total protein analysis was performed using Image $\mathbf{J}$ software.

\section{RNA interference}

The small interfering RNAs (siRNAs), which targets mTOR, were purchased from RIBOBIO (China). The sequences of siRNAs are listed in Table S2. We transfected the siRNAs into cells using Lipofectamine 3000 Reagent (Invitrogen) according to the manufacturer's instructions.

\section{In vivo studies}

\section{Ethics statement}

All experimental protocols and animal experiments were approved by the Animal Care and Use Committee of Sun Yat-sen University and conducted in accordance with the National Institutes of Health Guide for the Care and Use of Laboratory Animals.

\section{In vivo bioluminescence imaging}

In vivo bioluminescence imaging was used to evaluate the retention of exos in the mouse model. Mice were euthanized with isoflurane before they were imaged using the non-invasive In-Vivo FX Pro (Bruke) imaging system.

\section{In vivo degradation and biocompatibility of the hydrogels}

Adult male C57BL/6J mice (6-8 weeks old, $\mathrm{n}=27$ ) were used for the evaluation of in vivo biodegradability and biocompatibility of each hydrogel. The mice were divided into GM hydrogel $(n=9)$, GMP hydrogel (n=9) and GMPE hydrogel (n=9) groups. Each hydrogel was subcutaneously inserted into the backs of mice, which were sacrificed at weeks 1, 3, and 6 after implantation. The surrounding tissue was removed and fixed in $4 \%$ paraformaldehyde for further pathological analysis with hematoxylin and eosin (HE). The thickness of the fibrotic capsule was determined using Image J software.

\section{Animals spinal cord hemisection model}


Seventy-five adult male C57BL/6J mice (6-8 weeks old) were randomly assigned to sham $(\mathrm{n}=15)$, SCI ( $n=15)$, GM $(n=15)$, GMP $(n=15)$ and GMPE $(n=15)$ groups. Animals were anesthetized by intraperitoneal injection of a mixture of $5 \mathrm{mg} / \mathrm{kg}$ xylazine and $70 \mathrm{mg} / \mathrm{kg}$ ketamine. A $2 \mathrm{~mm}$ long longitudinal right-side spinal cord hemisection was performed at the T9-10 level under a microscope. Then, hydrogels were transplanted into the lesion sites, which were then surgically closed. Mice bladder were emptied manually twice daily until spontaneous voiding resumed.

\section{Functional recovery and footprint analysis in SCI model mice}

Locomotion recovery in the mice was evaluated using the Basso mouse scale (BMS) scores and footprint analysis 6 weeks after SCI. In order to assure unbiased evaluation of behavioral recovery, the behavioral recovery of mice was assessed by an independent experimenter. BMS scores were performed to assess mice hind limb behavior before the operation and then weekly after SCI. Mice were allowed to walk freely on a grid and the scores were judged based on hind limb movement function ranging from 0 (no ankle movement) to 9 (complete function recovery). Footprint analysis was applied to assess body weight support and physical coordination. The fore and hind limbs of the mice were dipped into blue and red ink, respectively. Then, the mice were allowed to walk on a paper-covered narrow surface. The distance between the two sides of the hind paws was identified as the base of support. Stride length was characterized as the perpendicular distance between the fore and hind limbs to assess the coordination ability. The angle which formed by two lines crossing the center of the hind paw to the third toe, and the stride line was defined as the angle of rotation. The frequency of toe dragging was characterized as the ratio of dragging to total footsteps.

\section{Spinal evoked compound muscle action potentials (CMAPs)}

The CMAPs data were recorded with a BIOPAC MP160 system (BIOPAC) from mice 6 weeks after SCI. Mice were anesthetized by intraperitoneal injection of a $5 \mathrm{mg} / \mathrm{kg}$ xylazine and 70 $\mathrm{mg} / \mathrm{kg}$ ketamine mixture before their limbs were fixed on a board with cloth bands. Two stimulating electrodes were inserted into the spinal cord above the injury site, and CMAPs were used to evaluate motion pathways from recording electrodes inserted into the ipsilateral gastrocnemius. A ground electrode was inserted into the mouse tail. The stimulation strength was $0.5 \mathrm{~V}$ and the stimulus duration was $500 \mathrm{~ms}$.

\section{In vivo magnetic resonance imaging (MRI)}

Mice were anesthetized by suction anesthesia and mounted in the supine position within a 7.0Tesla MR scanner (PharmaScan70/16 US, Bruker Biospin MRI GmbH) configured with a dedicated animal coil. Conventional MRI scans were performed at 3 and 6 weeks after surgery. For conventional MRI, a sagittal T2-weighted image (T2WI) and a coronal T2-weighted image (T2WI) were obtained. A spin echo (SE) sequence with the following parameters was used to acquire anatomical images: sagittal T2WIs (time of repetition (TR) /time of echo (TE) 1263 $\mathrm{ms} / 25 \mathrm{~ms}, 512 \times 512$ matrix, field of view (FOV) 16, slice thickness $0.5 \mathrm{~mm}$ ) and coronal T2WIs (TR/TE $1263 \mathrm{~ms} / 25 \mathrm{~ms}, 512 \times 512$ matrix, FOV 16, slice thickness $1.0 \mathrm{~mm}$ ).

\section{Hemocompatibility of the hydrogels}


In vitro hemolysis analysis was used to evaluate the hemocompatibility of the hydrogels. Blood samples were collected in anticoagulation tubes and co-cultured with each hydrogel in $37{ }^{\circ} \mathrm{C}$ water for $4 \mathrm{~h}$. PBS and Triton-100X were set as negative and positive group, respectively. After centrifugation at $10000 \mathrm{~g}$ for $5 \mathrm{~min}$ at $4{ }^{\circ} \mathrm{C}$, the supernatant was transferred to 96 -well plates and measured using an enzyme-labeling instrument (SpectraMax M5, $540 \mathrm{~nm}$ ). The hemolysis percentage was calculated using the following equation:

\section{Histological analysis}

$$
\text { Hemolysis }(\%)=\frac{\text { Sample absorbance }- \text { Negative control }}{\text { Positive absorbance }- \text { Negative control }} \times 100
$$

Mice were deep euthanized using $0.6 \%$ sodium pentobarbital $(10 \mathrm{~g} / 0.1 \mathrm{~mL}$, Merck) 6 weeks postSCI. The animals were then intracardially perfused with PBS followed by $4 \%$ paraformaldehyde. The spinal cord containing the injury site was dissected, fixed in $4 \%$ paraformaldehyde for $24 \mathrm{~h}$, and embedded in paraffin. Samples were then cut into $20-\mu \mathrm{m}$ thick sections using a Leica RM2245 electric slicer. A general review and lesion cavity assessment was performed with hematoxylin-eosin (HE) staining in samples from each spinal cord treatment group. Luxol fast blue (LFB) staining was used to identify remyelination at the lesion sites.

\section{Statistical analysis}

All values are represented as the mean \pm standard deviation (SD). Statistical analysis was performed using Statistical Product and Service Solutions software (SPSS, version 22.0). Repeated-measures one-way analysis of variance (ANOVA) followed by Bonferroni's multiple comparison test was used to compare differences between treatment groups. Differences were considered statistically significant at $\mathrm{p}<0.05$.

\section{H2: Supplementary Materials}

Supplementary material for this article is available at $\mathrm{XXX}$

Fig. S1. Identification of BMSCs and BMSC-exos.

Fig. S2. Physical properties of different hydrogels.

Fig. S3. In vitro and in vivo exos release from loaded hydrogels.

Fig. S4. In vitro biocompatibility of hydrogels.

Fig. S5. In vivo biocompatibility and biodegradability of implanted hydrogels.

Fig. S6. Microglia M1/M2 polarization was evaluated after $\mathrm{p}-\mathrm{I} \kappa \mathrm{B} \alpha$ was inhibited with BAY 117082 treatment.

Fig. S7. NSC axon outgrowth on GMPE hydrogels after selective p-mTOR inhibition.

Fig. S8. Spinal cord pathology after SCI at 3 and 6 weeks.

Fig. S9. GMPE hydrogel suppressed early inflammation after SCI.

Table S1. Primer sequences of each gene was showed below.

Table S2. Sequences of three siRNAs were showed below.

\section{References and Notes}

[1] E.J. Bradbury, E.R. Burnside, Moving beyond the glial scar for spinal cord repair, Nat Commun 10(1) (2019) 3879. 
[2] H. Ren, X. Chen, M. Tian, J. Zhou, H. Ouyang, Z. Zhang, Regulation of Inflammatory Cytokines for Spinal Cord Injury Repair Through Local Delivery of Therapeutic Agents, Advanced Science 5(11) (2018) 1800529.

[3] J. Chedly, S. Soares, A. Montembault, Y.V. Boxberg, F. Nothias, Physical chitosan microhydrogels as scaffolds for spinal cord injury restoration and axon regeneration, Biomaterials 138 (2017) 91.

[4] J. Park, D. JT, M. DJ, S. DR, C. BJ, A. AJ, S. LD, Local Immunomodulation with Antiinflammatory Cytokine-Encoding Lentivirus Enhances Functional Recovery after Spinal Cord Injury, Molecular Therapy the Journal of the American Society of Gene Therapy 26(7) (2018) 1756.

[5] C.S. Ahuja, J.R. Wilson, S. Nori, M.R.N. Kotter, C. Druschel, A. Curt, M.G. Fehlings, Traumatic spinal cord injury, Nat Rev Dis Primers 317018.

[6] B. Zhang, W. Yan, Y. Zhu, W. Yang, W. Le, B. Chen, R. Zhu, L. Cheng, Nanomaterials in Neural-Stem-Cell-Mediated Regenerative Medicine: Imaging and Treatment of Neurological Diseases, Advanced Materials 30(17) (2018) 1705694.

[7] F. Lei, C. Liu, X. Chen, Directing Induced Pluripotent Stem Cell Derived Neural Stem Cell Fate with a Three-Dimensional Biomimetic Hydrogel for Spinal Cord Injury Repair, ACS Applied Materials \& Interfaces 10(21) (2018) 17742-17755. [8] L.T.A. Hong, Y.-M. Kim, H.H. Park, D.H. Hwang, Y. Cui, E.M. Lee, S. Yahn, J.K. Lee, S.-C. Song, B.G. Kim, An injectable hydrogel enhances tissue repair after spinal cord injury by promoting extracellular matrix remodeling, Nature Communications 8(1) (2017) 533.

[9] J. Koffler, W. Zhu, X. Qu, O. Platoshyn, J.N. Dulin, J. Brock, L. Graham, P. Lu, J. Sakamoto, M. Marsala, S. Chen, M.H. Tuszynski, Biomimetic 3D-printed scaffolds for spinal cord injury repair, Nat Med 25(2) (2019) 263-269.

[10] B. Guo, P.X. Ma, Conducting Polymers for Tissue Engineering, Biomacromolecules 19(6) (2018) 1764-1782.

[11] Y. Wu, Y.X. Chen, J. Yan, D. Quinn, P. Dong, S.W. Sawyer, P. Soman, Fabrication of conductive gelatin methacrylate-polyaniline hydrogels, Acta Biomaterialia 33 (2016) 122-130. [12] H. Lu, N. Zhang, M. Ma, Electroconductive hydrogels for biomedical applications, Wiley Interdisciplinary Reviews Nanomedicine and Nanobiotechnology (21) (2019) e1568. [13] A. Burnstine-Townley, Y. Eshel, N. Amdursky, Conductive Scaffolds for Cardiac and Neuronal Tissue Engineering: Governing Factors and Mechanisms, Advanced Functional Materials 2020, 30, 1901369.

[14] L. Zhou, L. Fan, X. Yi, Z. Zhou, C. Liu, R. Fu, C. Dai, Z. Wang, X. Chen, P. Yu, Soft Conducting Polymer Hydrogels Cross-Linked and Doped by Tannic Acid for Spinal Cord Injury Repair, ACS Nano 12(11) (2018) 10957.

[15] G. Radley, I.L. Pieper, S. Ali, F. Bhatti, C.A. Thornton, The Inflammatory Response to Ventricular Assist Devices, Frontiers in immunology 9 (2018) 2651.

[16] K. English, A. French, K.J. Wood, Mesenchymal stromal cells: facilitators of successful transplantation. Cell stem cell 7(4) (2010) 431-42.

[17] C. Frühbeis, D. Fröhlich, E.-M. Krämer-Albers, Emerging Roles of Exosomes in NeuronGlia Communication, Frontiers in Physiology 30 (3) (2012) 119. 
[18] M.M. Holm, J. Kaiser, M.E. Schwab, Extracellular Vesicles: Multimodal Envoys in Neural Maintenance and Repair, Trends in Neurosciences 41 (6) (2018) 360-372.

[19] S. Guo, N. Perets, O. Betzer, S. Ben-Shaul, A. Sheinin, I. Michaelevski, R. Popovtzer, D. Offen, S. Levenberg, Intranasal Delivery of Mesenchymal Stem Cell Derived Exosomes Loaded with Phosphatase and Tensin Homolog siRNA Repairs Complete Spinal Cord Injury, ACS Nano 13(9) (2019) 10015-10028.

[20] X. Ouyang, X. Han, Z. Chen, J. Fang, X. Huang, H. Wei, MSC-derived exosomes ameliorate erectile dysfunction by alleviation of corpus cavernosum smooth muscle apoptosis in a rat model of cavernous nerve injury, Stem Cell Research \& Therapy 9(1) (2018). [21] R.A. Green, N.H. Lovell, G.G. Wallace, L.A. Poole-Warren, Conducting polymers for neural interfaces: challenges in developing an effective long-term implant, Biomaterials 29(24-25) (2008) 3393-9.

[22] M. Nahrendorf, F.K. Swirski, Abandoning M1/M2 for a Network Model of Macrophage Function, Circulation research 119(3) (2016) 414-7.

[23] S. Jiao, Y. Liu, Y. Yao, J. Teng, miR-124 promotes proliferation and differentiation of neuronal stem cells through inactivating Notch pathway, Cell \& Bioscience 7(1) (2017) 68. [24] Z. Yang, A. Zhang, H. Duan, S. Zhang, P. Hao, K. Ye, Y.E. Sun, X. Li, NT3-chitosan elicits robust endogenous neurogenesis to enable functional recovery after spinal cord injury, Proc Natl Acad Sci U S A 112(43) (2015) 13354-13359.

[25] W. Zhao, L. Glavas, K. Odelius, U. Edlund, A.C. Albertsson, Facile and Green Approach towards Electrically Conductive Hemicellulose Hydrogels with Tunable Conductivity and Swelling Behavior, Chemistry of Materials 26(14) (2014) 4265-4273.

[26] C.J. Bettinger, J.P. Bruggeman, M. Asish, J.T. Borenstein, L. Robert, Biocompatibility of biodegradable semiconducting melanin films for nerve tissue engineering, Biomaterials 30(17) (2009) 3050-3057.

[27] R. Zhu, Z. Sun, C. Li, S. Ramakrishna, K. Chiu, L. He, Electrical stimulation affects neural stem cell fate and function in vitro, Exp Neurol 319 (2019) 112963.

[28] J.-S. Rao, C. Zhao, A. Zhang, H. Duan, P. Hao, R.-H. Wei, J. Shang, W. Zhao, Z. Liu, J. Yu, K.S. Fan, Z. Tian, Q. He, W. Song, Z. Yang, Y.E. Sun, X. Li, NT3-chitosan enables de novo regeneration and functional recovery in monkeys after spinal cord injury, Proc Natl Acad Sci U S A 115(24) (2018) E5595-E5604.

[29] H.O.B. Gautier, K.A. Evans, K. Volbracht, R. James, S. Sitnikov, I. Lundgaard, F. James, C. Lao-Peregrin, R. Reynolds, R.J.M. Franklin, Neuronal activity regulates remyelination via glutamate signalling to oligodendrocyte progenitors, Nature Communications 6 (2015) 8518. [30] D. Veber, Why does remyelination fail in multiple sclerosis?, Nature Reiview Neuroscience 3(9) (2002) 705-714.

[31] P. Stathopoulos, H. Alexopoulos, M.C. Dalakas, Autoimmune antigenic targets at the node of Ranvier in demyelinating disorders, Nature Reviews Neurology 11(3) (2015) 143-56. [32] D. Ma, Y. Zhao, L. Huang, Z. Xiao, B. Chen, Y. Shi, H. Shen, J. Dai, A novel hydrogelbased treatment for complete transection spinal cord injury repair is driven by microglia/macrophages repopulation, Biomaterials 237 (2020) 119830-119830. 
[33] S. David, A. Kroner, Repertoire of microglial and macrophage responses after spinal cord injury, Nature Reviews Neuroscience 12(7) (2011) 388.

[34] C.S. Chamberlain, A.B. Clements, J.A. Kink, U. Choi, G.S. Baer, M.A. Halanski, P. Hematti, R. Vanderby, Extracellular vesicle-educated macrophages promote early Achilles tendon healing, STEM CELLS 37 (2019) 652-662.

[35] L.X. Zhang, Y.M. Yin, Z.Q. Zhang, L.X. Deng, Grafted bone marrow stromal cells: a contributor to glial repair after spinal cord injury, The Neuroscientist: a review journal bringing neurobiology, neurology and psychiatry 21(3) (2015) 277-89.

[36] M. Ghibaudi, M. Boido, A. Vercelli, Functional integration of complex miRNA networks in central and peripheral lesion and axonal regeneration, Progress in Neurobiology 158 (2017) S0301008217300126.

[37] A. Salminen, J. Huuskonen, J. Ojala, A. Kauppinen, K. Kaarniranta, T. Suuronen, Activation of innate immunity system during aging: NF-kB signaling is the molecular culprit of inflammaging, Ageing research reviews 7(2) (2008) 83-105.

[38] H. Lu, N. Zhang, M. Ma, Electroconductive hydrogels for biomedical applications, Wiley interdisciplinary reviews. Nanomedicine and nanobiotechnology 11(6) (2019) e1568.

[39] A.R. Spencer, E. Shirzaei Sani, J.R. Soucy, C.C. Corbet, A. Primbetova, R.A. Koppes, N. Annabi, Bioprinting of a Cell-Laden Conductive Hydrogel Composite, ACS applied materials \& interfaces 11(34) (2019) 30518-30533.

[40] Y.S. Jeong, W.K. Oh, S. Kim, J. Jang, Cellular uptake, cytotoxicity, and ROS generation with silica/conducting polymer core/shell nanospheres, Biomaterials 32(29) (2011) 7217-7225.

\section{Acknowledgments}

Funding: This work was supported by the National Key R\&D Program of China (Nos. 2018YFC1105304 and 2018YFC1105301), the National Natural Science Foundation of China (Nos. 51932002, 51903087, 51772105, 51772106, and 31771080), and the Natural Science Foundation of Guangdong Province (No. 2020A1515011369) Author contributions: Lei Fan designed and synthesized the GMPE hydrogel under the guidance of Lei Zhou and Chengyun Ning. Lei Fan, Can Liu, and Xiuxing Chen performed the in vitro and in vivo experiments. Lei Fan analyzed the data and wrote the manuscript with guidance from Chengyun Ning, Lei Zhou, and Qiyou Wang. Peng Yu and Guoxin Tan provided experinmental guidance. Yan Zou and Huiquan Wen helped optimize device applications, especially the mouse MRI. Yian Luo and Fang Lu conducted the experimental verification. Chengyun Ning, Lei Zhou and Qiyou Wang provided guidance during all stages of the project. Competing interests: The authors declare that they have no competing interests. Data and materials availability: All data needed to evaluate the conclusions in this paper are presented in the paper and/or the Supplementary Materials. Additional data related to this paper may be requested from the authors. 
A
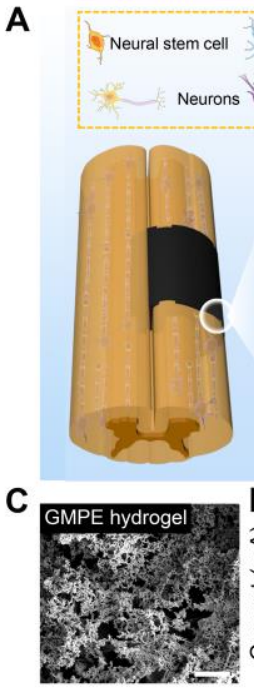

D

Neurogenesis Myelin-associated Inflammatio
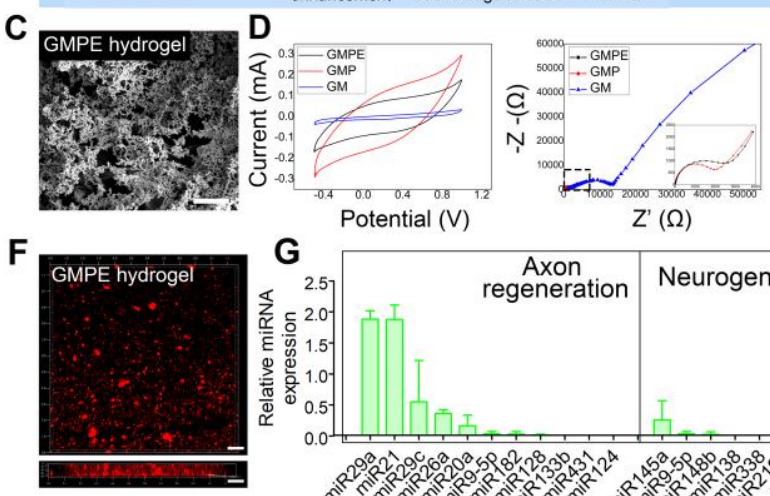

I
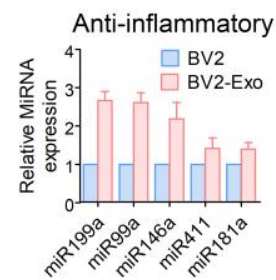

$\mathbf{J}$

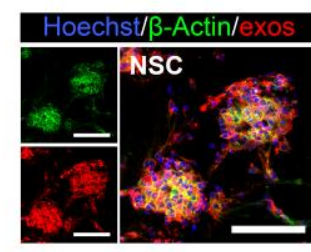

B
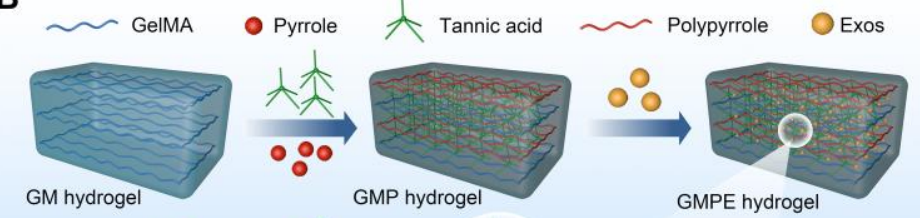

GMP hydrogel

GMPE hydrogel
1024

1025

1026

1027

1028

1029

1030

1031

1032

1033

1034

1035

1036

1037

1038

1039

1040

1041

1042

1043

1044

1045

Fig. 1 Characteristics of the GMPE hydrogels. (A) Illustration of how the GMPE hydrogel can reduce early inflammation, enhance neurogenesis and promote myelin-associated axonal regrowth to synergistically promote locomotor recovery after spinal cord hemisection. (B) The three-step synthesis procedure for the GMPE hydrogel was illustrated. The GMP hydrogel was synthesized by TA interacting with the amide bond on the GM backbone and the nitrogen groups on PPy chains. BMSC-exos were reversibly immobilized into GMP hydrogels via hydrogen bond formation between TA polyphenol groups and phosphate groups in exos phospholipid to form GMPE hydrogel. (C) Micro-structure of the GMPE hydrogel was observed by SEM. Scale bars, $25 \mu \mathrm{m}$. (D) Electrical characterization, including cyclic voltammograms (CV), electrochemical impedance (EIS), current-voltage (I-V) and Bode plots of GMP and GMPE hydrogels showed excellent electrical performance. (E) After the isolation of transected spinal cords, the stimulating electrical signals were retransmitted by GMPE hydrogels. (F) IF imaging showed that exos were evenly distributed into the GMPE hydrogel and the penetration depth of the exosomes was more than $100 \mu \mathrm{m}$. Scale bars, $100 \mu \mathrm{m}$. (G) RT-qPCR indicated that BMSC-exos express of axon regeneration-related, neurogenesis-related, and anti-inflammatory-related miRNAs $(n=3)$. $(H)$ BV2 cells cultured on the GMPE hydrogel can normally phagocytize exos released from the hydrogel. Scale bars, $100 \mu \mathrm{m}$. (I) Anti-inflammatory-related miRNAs expression increased as the result of BV2 cells phagocytosing exos $(n=3)$. (J) PKH26 labeled exos were clearly detected in the cytoplasm of NSCs, suggesting successful in vitro endocytosis of exos released from the GMPE hydrogel. Scale bars, $100 \mu \mathrm{m}$. (K) Axon regeneration-related and neurogenesis-related miRNAs expression increased after NSCs phagocytize exos $(n=3)$. 


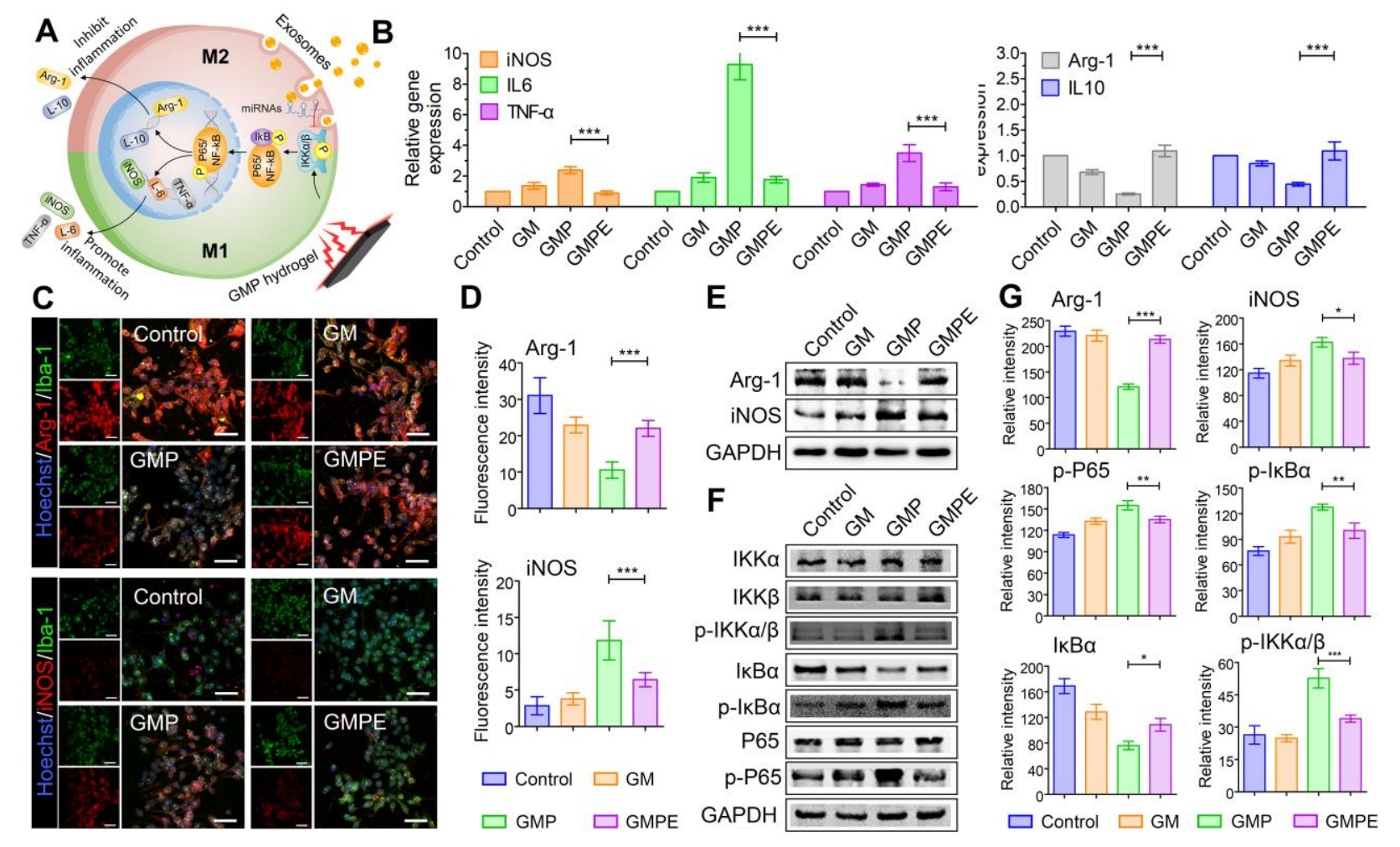

Fig. 2 GMPE hydrogels promote microglia M1 to M2 switch by activating the NF-кB pathway. (A) An illustration of microglia switching from an M1- to M2-dominant phenotype through NF- $\kappa \mathrm{B}$ pathway activity. (B) RT-qPCR results of the level gene expression of proinflammatory factors Arg-1 and IL-10 and the anti-inflammatory factors iNOS, IL-6 and TNF- $\alpha$ in BV2 cells cultured on hydrogels $(n=3)$. Statistical differences were determined using an ANOVA with Bonferroni's multiple comparison test. (C) IF imaging showing the proportion of Arg-1 positive and iNOS positive BV2 cells cultured in each hydrogel treatment group. Green IF represents the microglia/macrophage specific protein marker Iba-1, red fluorescence represents the M1/M2 microglia/macrophage phenotype marker iNOS or Arg-1, blue fluorescence represents the nuclear marker Hoechst 33342. Scale bars, $200 \mu \mathrm{m}$. (D) Quantification of fluorescence intensity of iNOS and Arg-1 level in each hydrogel treatment group ( $n=5)$. Statistical differences were determined using ANOVA with Bonferroni's multiple comparison test. (E) GMPE hydrogel promoted BV2 cell M1 polarization. (F) GMPE hydrogel up-regulated the expression of Arg-1, iNOS, and relative proteins of NF- $\kappa \mathrm{B}$ pathway, further indicating that the GMPE hydrogel promotes BV2 cell M1 polarization through NF- $\kappa$ B pathway activation. (G) Protein band intensity was quantified using Image $J(n=3)$. Statistical differences between gene expression and protein expression were determined using an ANOVA with Bonferroni's multiple comparison test $(* \mathrm{p}<0.05, * * \mathrm{p}<0.01, * * * \mathrm{p}<0.001)$. 


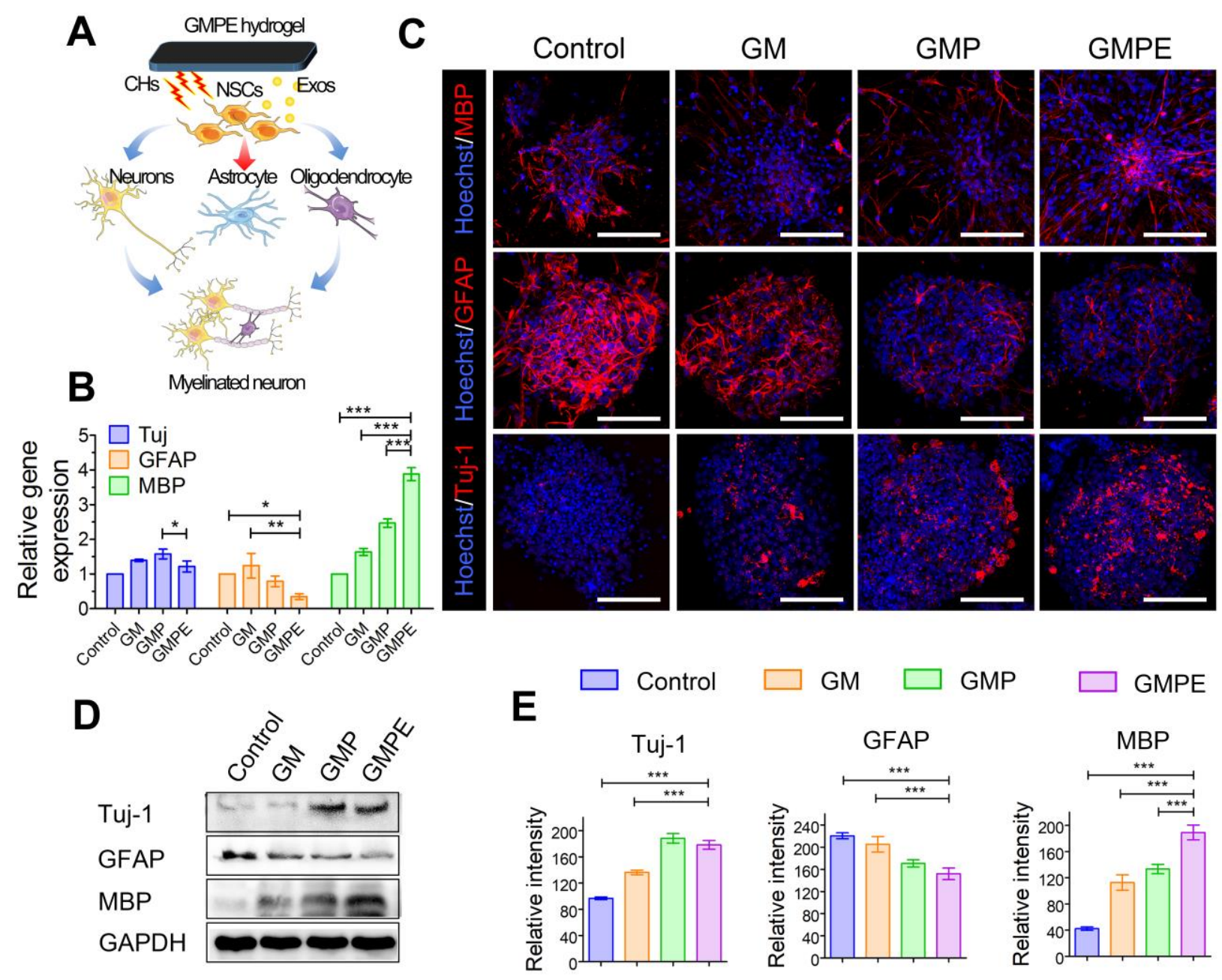

Fig. 3 NSCs differentiation on hydrogels. (A) An illustration showing that the GMPE hydrogel promotes NSC neuron and oligodendrocyte differentiation to form myelinated neurons, but inhibited astrocyte differentiation. (B) The RT-qPCR data show GMPE promote MBP expression $(n=3)$. Statistical differences were determined using ANOVA with Bonferroni's multiple comparison test. (C) IF imaging of NSC neuron, astrocyte, and oligodendrocyte differentiation after 7 days on control or hydrogel conditions. Red IF represents the neuron marker Tuj-1, astrocyte marker GFAP, or oligodendrocyte marker MBP, respectively. Scale bars, $100 \mu \mathrm{m}$. (D) WB analysis detected Tuj-1, GFAP, and MBP protein expression in NSCs cultured on hydrogels and control conditions for 7 days. (E) Protein band intensity was quantified $(n=3)$ and analyzed using an ANOVA with Bonferroni's multiple comparison test $(* \mathrm{p}<0.05, * * \mathrm{p}<0.01, * * * \mathrm{p}<0.001)$. 

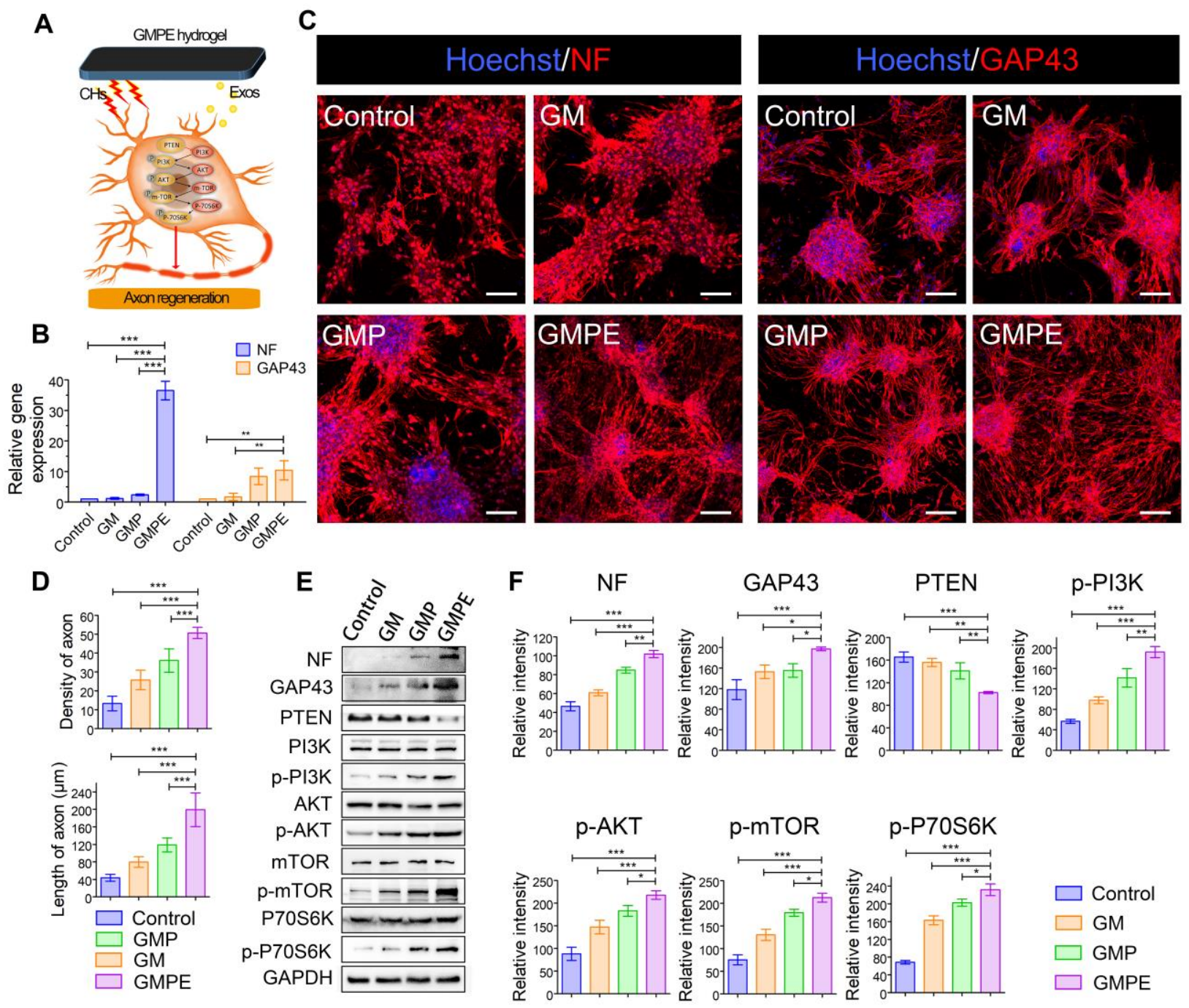

Fig. 4 Axon outgrowth on hydrogels. (A) Illustration of the BMSC-exos conductive hydrogel mechanism and synergistic promotion of axon outgrowth through the activation of the PTEN/PI3K/AKT/mTOR pathway. (B) RT-qPCR indicating that GMPE hydrogels can promote $\mathrm{NF}$ and GAP43 gene expression $(\mathrm{n}=3)$. Statistical differences determined using ANOVA with Bonferroni's multiple comparison test. (C) IF images of the axon-associated proteins NF and GAP43 in NSCs grown on hydrogels for 7 days. Red IF represents the NF or GAP43, respectively. Scale bars, $100 \mu \mathrm{m}$. (D) The density $(\mathrm{n}=5)$ and length of axons were quantified using Image $\mathrm{J}$ software $(n=11)$. Statistical differences were determined using an ANOVA with Bonferroni's multiple comparison test. (E) WB result of the expression of NF and GAP43 proteins and the relative protein expression of the PTEN/PI3K/AKT/mTOR pathway in NSCs cultured on control or hydrogel conditions for 7 days. (F) Protein band intensity was quantified $(n=3)$ and analyzed using an ANOVA with Bonferroni's multiple comparison test $(* \mathrm{p}<0.05, * * \mathrm{p}<0.01, * * * \mathrm{p}<0.001)$. 
A
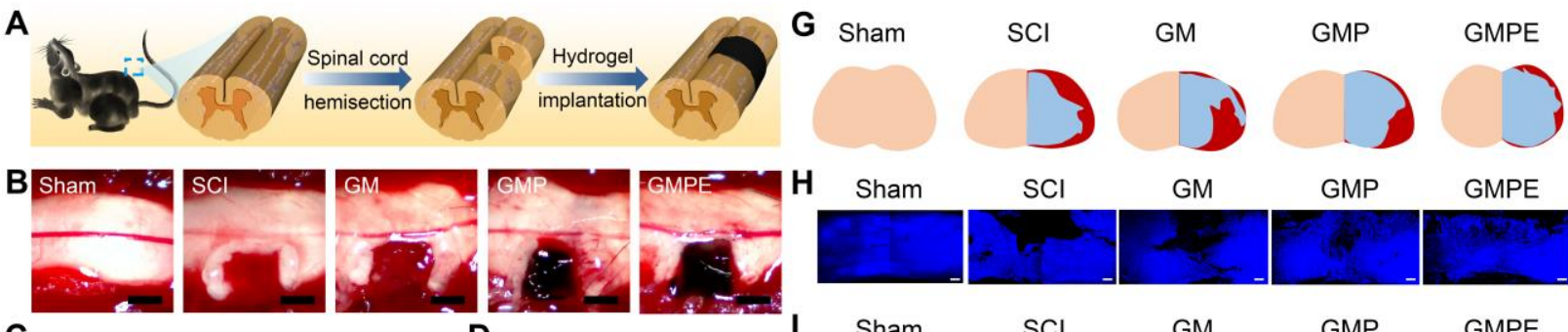

$\mathrm{SCl}$

GM

GMP

GMPE
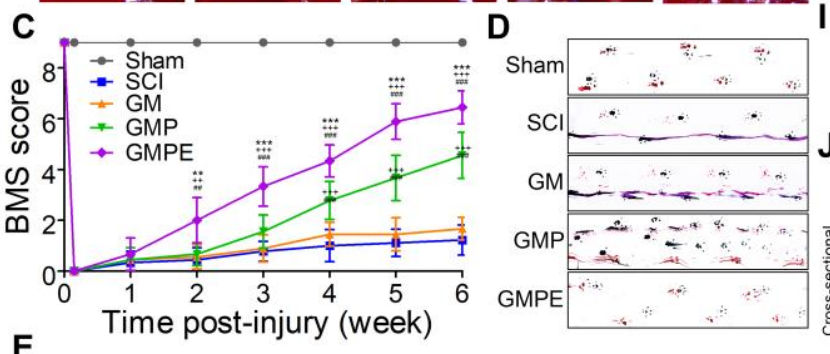

I Sham
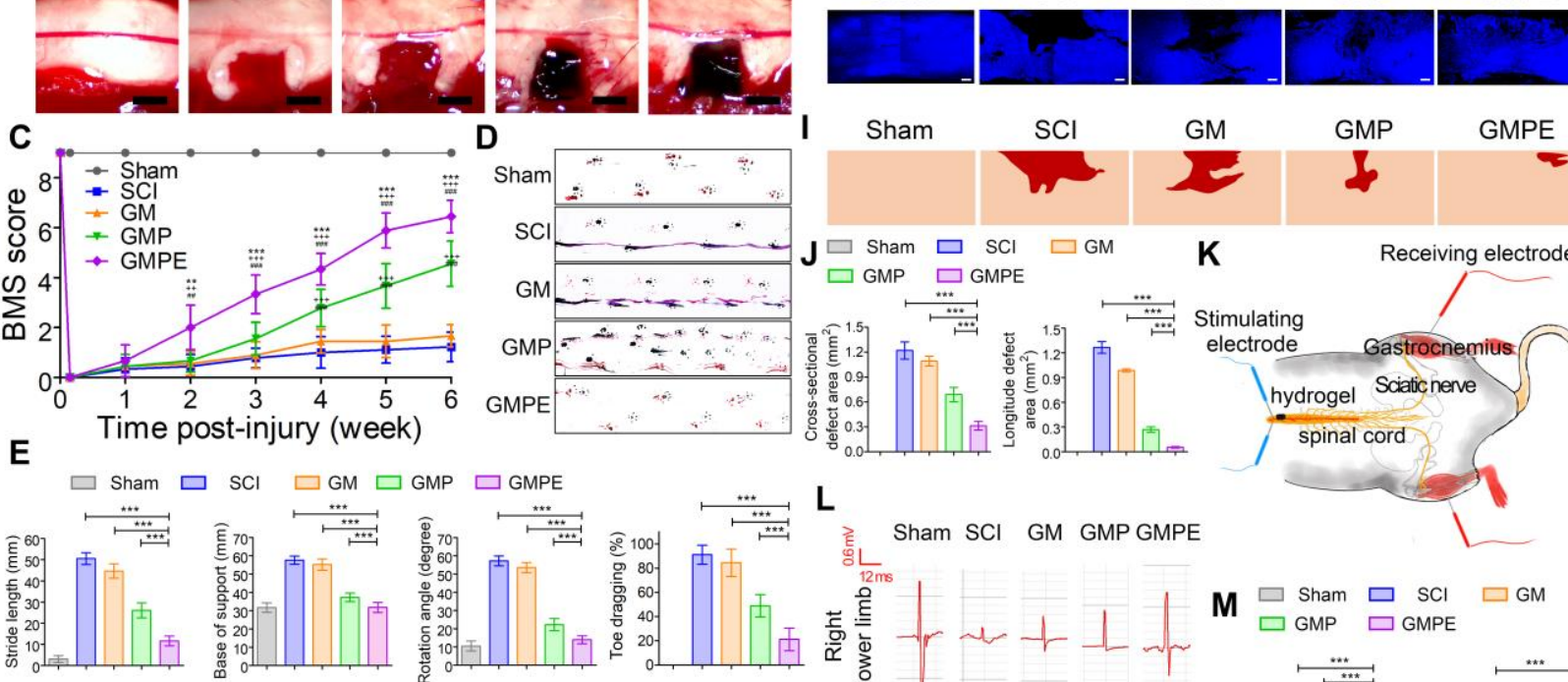

F Sham
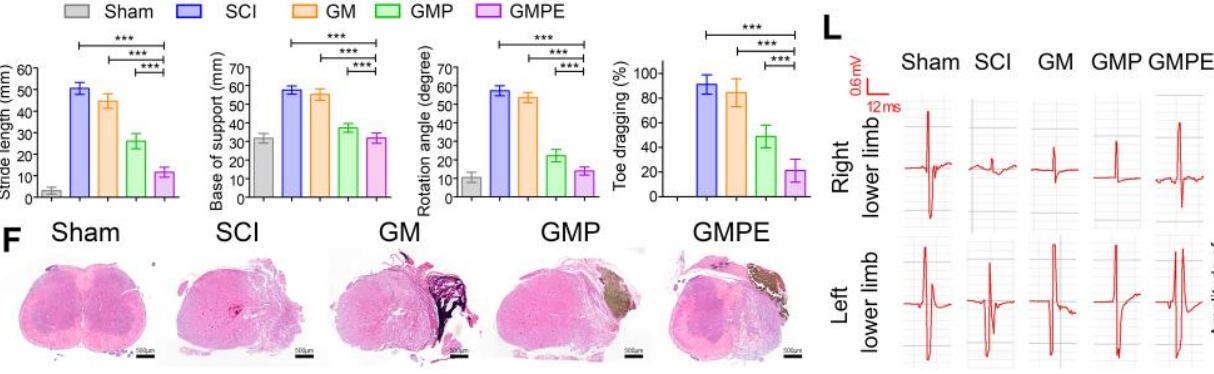

K

Receiving electrode

Stimulating
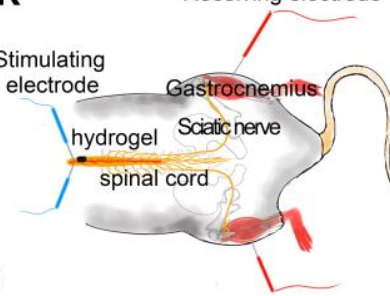

M Sham $\square \mathrm{SCl}$

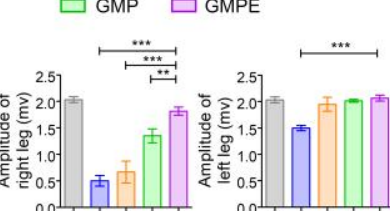

Fig. 5 Functional recovery of mice in different groups. (A) Illustration of the spinal cord hemisection and hydrogel implantation. (B) Different hydrogels were implanted at the cavitary site. Scale bars, $1 \mathrm{~mm}$. (C) Right hindlimb locomotor recovery in mice was evaluated using BMS scoring on a grid. Mice treated with the GMPE hydrogel had improved BMS locomotor scores 2 weeks after injury $(n=9)$ when compared to GMP $(* p<0.05, * * p<0.01, * * * p<0.001)$ and GM (+ $\mathrm{p}<0.05,++\mathrm{p}<0.01,+++\mathrm{p}<0.001)$ hydrogels, and $\mathrm{SCI}(\# \mathrm{p}<0.05, \# \# \mathrm{p}<0.01$, \#\#\# $\mathrm{p}<0.001)$.

Statistical differences were determined using an ANOVA with Bonferroni's multiple comparison test. (D) Representative footprints used to analyze recovery of hindlimb motor function. The forelimb footprints are shown in blue, and the hindlimb footprints in red. (E) Stride length, base of support, rotation angle and toe dragging were used to quantify the recovery of locomotion at 6 weeks after injury $(n=9)$. Statistical differences were determined using an ANOVA with Bonferroni's multiple comparison test. (F) HE staining showing the morphology of transverse spinal cord sections of sham and hydrogel implantation after SCI. (G) Representative reconstructions of the transverse spinal cord sections. Flesh-colored areas represent normal tissue, cavitary areas are highlighted in red, and blue-colored areas represent regenerated tissue. (H) IF images illustrating the morphology of longitudinal sections of the spinal cords. Scale bars, 200 $\mu \mathrm{m}$. (I) Representative images of reconstructed spinal cord longitudinal section. Flesh-colored areas represent normal tissue, while red-colored areas represent the cavitary areas. ( $\mathbf{J})$ Cavity volume of transverse spinal cord and longitudinal sections was quantified $(\mathrm{n}=3)$. Statistical differences were determined using an ANOVA with Bonferroni's multiple comparison test. (K) Illustration of the CMAP testing protocol in mice from GMPE hydrogel treatment group. (L) CMAP results from normal mice and those in different hydrogel implantation groups 6 weeks post-treatment. (M) Quantification of the CMAP amplitudes measured in mice from sham and hydrogel treatment groups $(n=3)$. Statistical differences were determined using an ANOVA with Bonferroni's multiple comparison test $\left(* \mathrm{p}<0.05,{ }^{* *} \mathrm{p}<0.01,{ }^{* * *} \mathrm{p}<0.001\right)$. 
GM

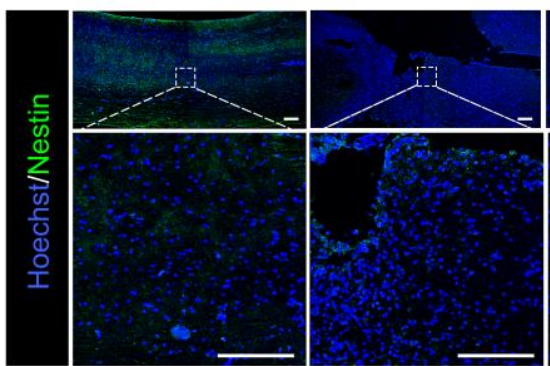

C

$\mathrm{SCl}$

GM
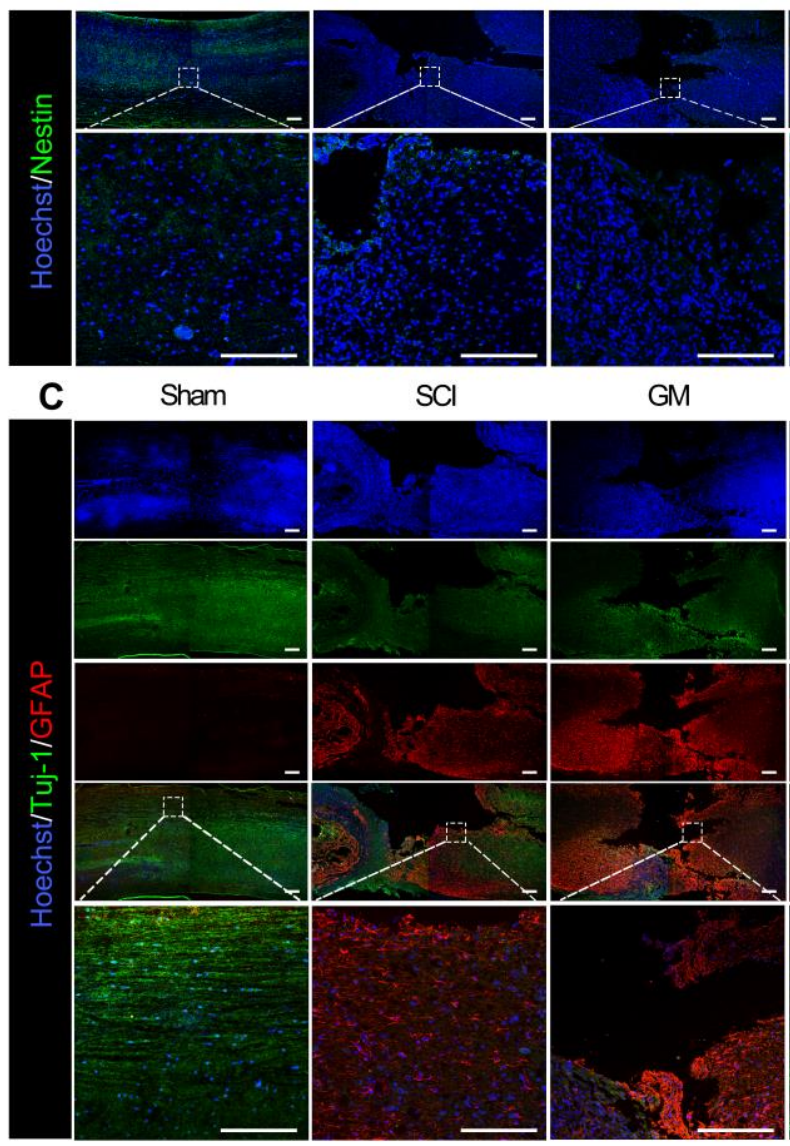

Caudal border $\mathbf{F}$

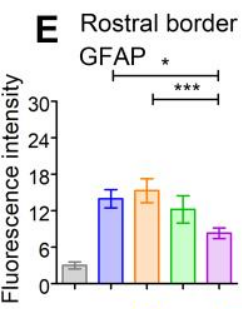

$\square$ Sham $\square \mathrm{SCl} \square \mathrm{GM} \square \mathrm{GMP} \square \mathrm{GMPE}$

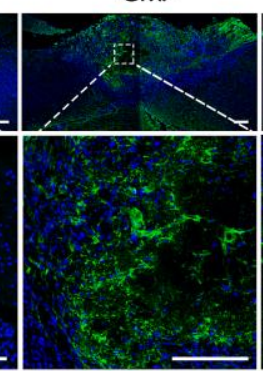

GMPE

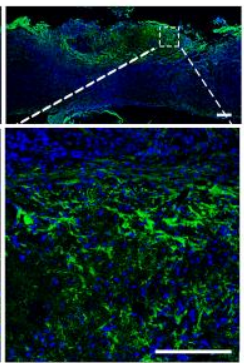

GMP
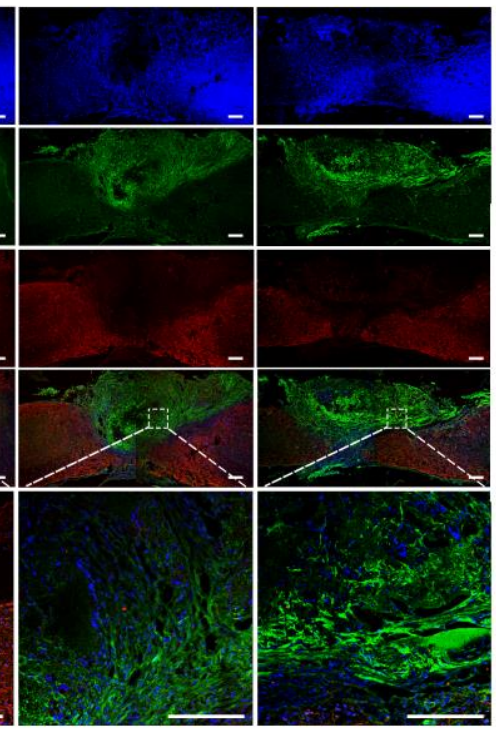

G Tuj-1

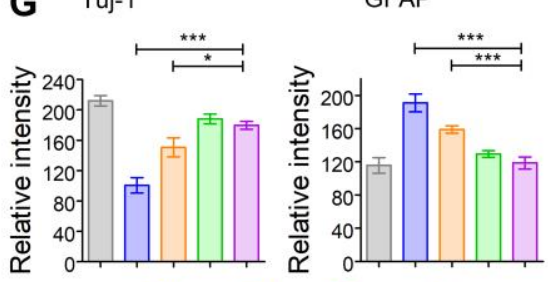

$\square \mathrm{Sham} \square \mathrm{SCl} \square \mathrm{GM} \square \mathrm{GMP} \square \mathrm{GMPE}$

Fig. 6 GMPE hydrogel implantation promotes endogenous neurogenesis. (A) Nestin-positive endogenous NSCs spontaneously migrated into the GMPE hydrogel 6 weeks after SCI. Green IF represents the NSC marker nestin. Scale bars, $200 \mu \mathrm{m}$. (B) Graph of the quantification of nestin IF intensity 6 weeks after SCI. The region of interest (ROI) was the injury site center $(n=3)$. Statistical differences were determined using an ANOVA with Bonferroni's multiple comparison test. (C) The GMPE hydrogel promotes endogenous NSC neuronal differentiation while inhibiting astrocytic differentiation in vivo. Green IF represents the neuronal marker Tuj-1. Red IF represents the astrocytic marker GFAP. Scale bars, $200 \mu \mathrm{m}$. (D) Graph of the Tuj-1 IF intensity quantification. The fluorescence intensity was measured at three ROIs: the rostral and caudal borders, and the injury site center $(n=3)$. Statistical differences were determined using an ANOVA with Bonferroni's multiple comparison test. (E) Graph of GFAP IF intensity quantification at same timepoint at the ROIs: rostral and caudal borders of the injury site $(n=3)$. Statistical differences were determined using an ANOVA with Bonferroni's multiple comparison test. (F) WB analysis of the neuronal and astrocytic differentiation protein markers in each treatment group. (G) Quantitative measurement of protein band intensity included calculating the statistical differences between samples $(n=3)$ using ANOVA with Bonferroni's multiple comparison test (* $^{*}$ $\mathrm{p}<0.05, * * \mathrm{p}<0.01, * * * \mathrm{p}<0.001)$. 


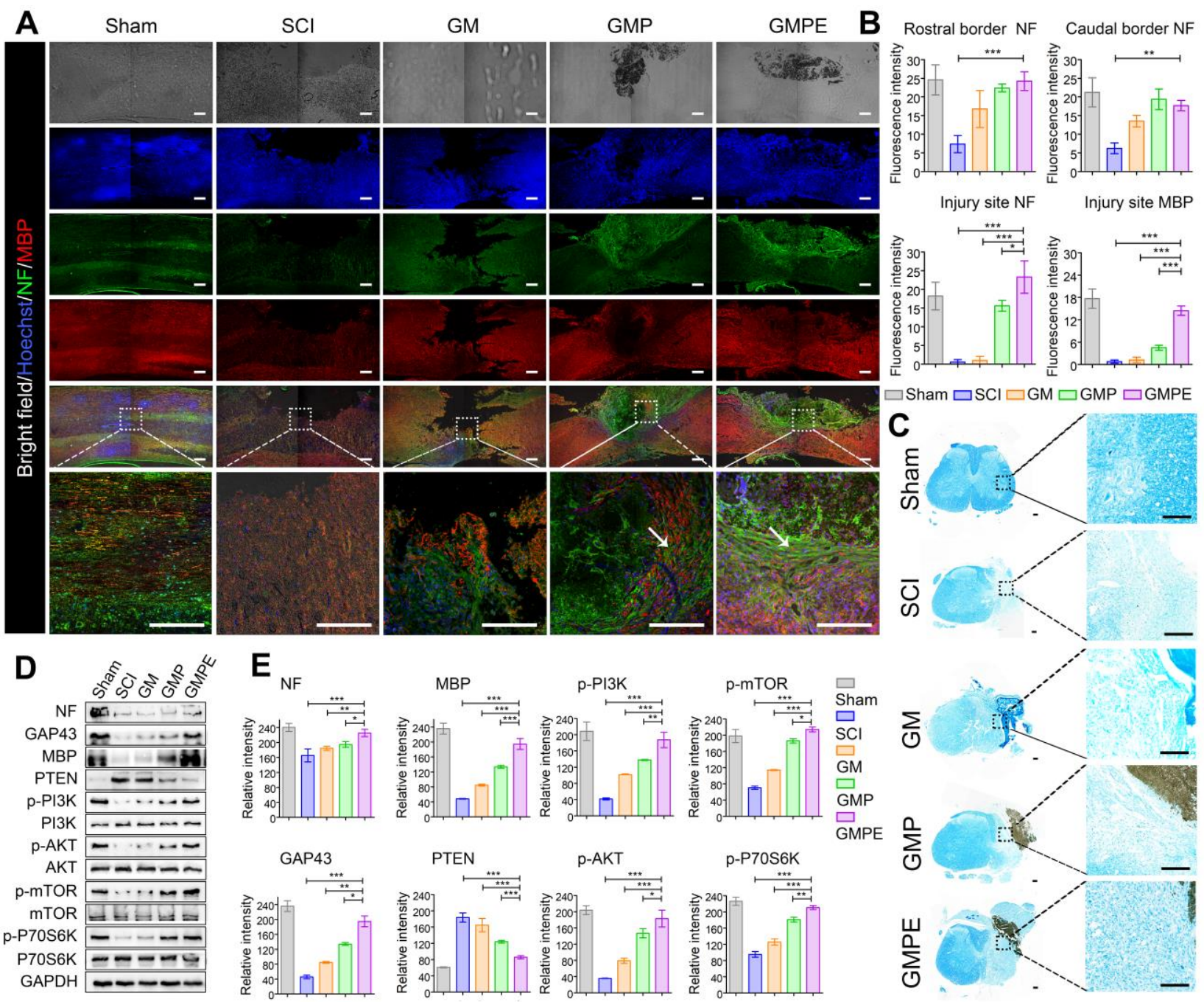

Fig. 7 GMPE hydrogel implantation promotes axon regeneration and remyelination in vivo. (A) Axonal regeneration and remyelination was evaluated in the five treatment groups 6 weeks post operation using IF imaging. Green IF represents the NF axon marker. Red IF represents the MBP oligodendrocyte marker. Scale bars, $200 \mu \mathrm{m}$. (B) Graph of NF and MBP IF intensity quantified from ROIs at both ends and the center of the injury site $(n=3)$. GFAP IF intensity ROI were measured from center of the injury site $(n=3)$. Statistical differences were determined using an ANOVA with Bonferroni's multiple comparison test. (C) Remyelination was evaluated by LFB staining which showed that nerve myelin was detected around the GMPE hydrogel. Scale bars, $200 \mu \mathrm{m}$. (D) Protein expression of NF, GAP43, MBP, and PTEN/PI3K/AKT/mTOR pathway proteins; GADPH was included as an expression control. (E) Graph of protein band intensity quantification $(n=3)$. Statistical differences were determined using an ANOVA with Bonferroni's multiple comparison test $(* \mathrm{p}<0.05, * * \mathrm{p}<0.01, * * * \mathrm{p}<0.001)$. 
A
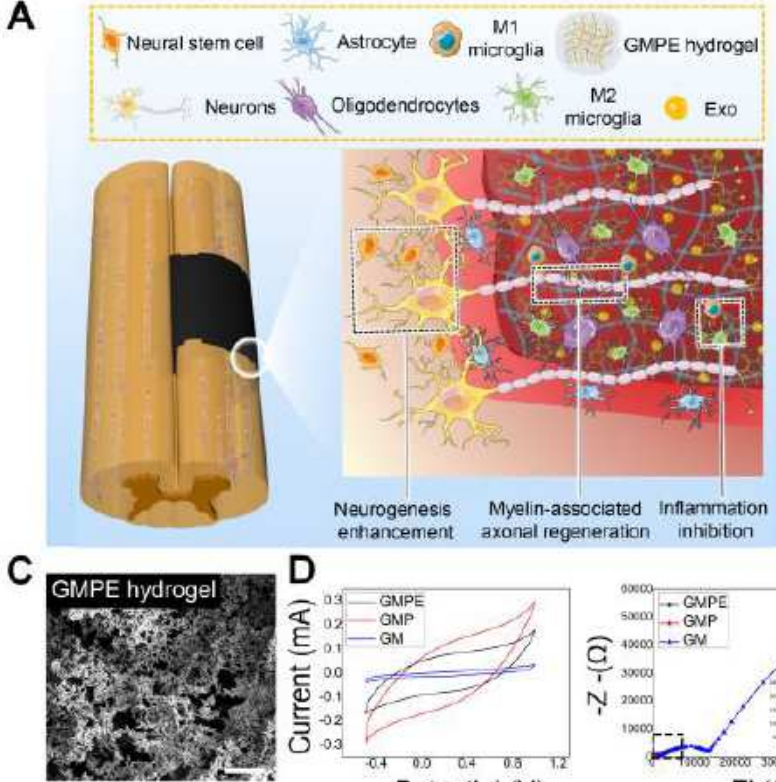

Potential (V)
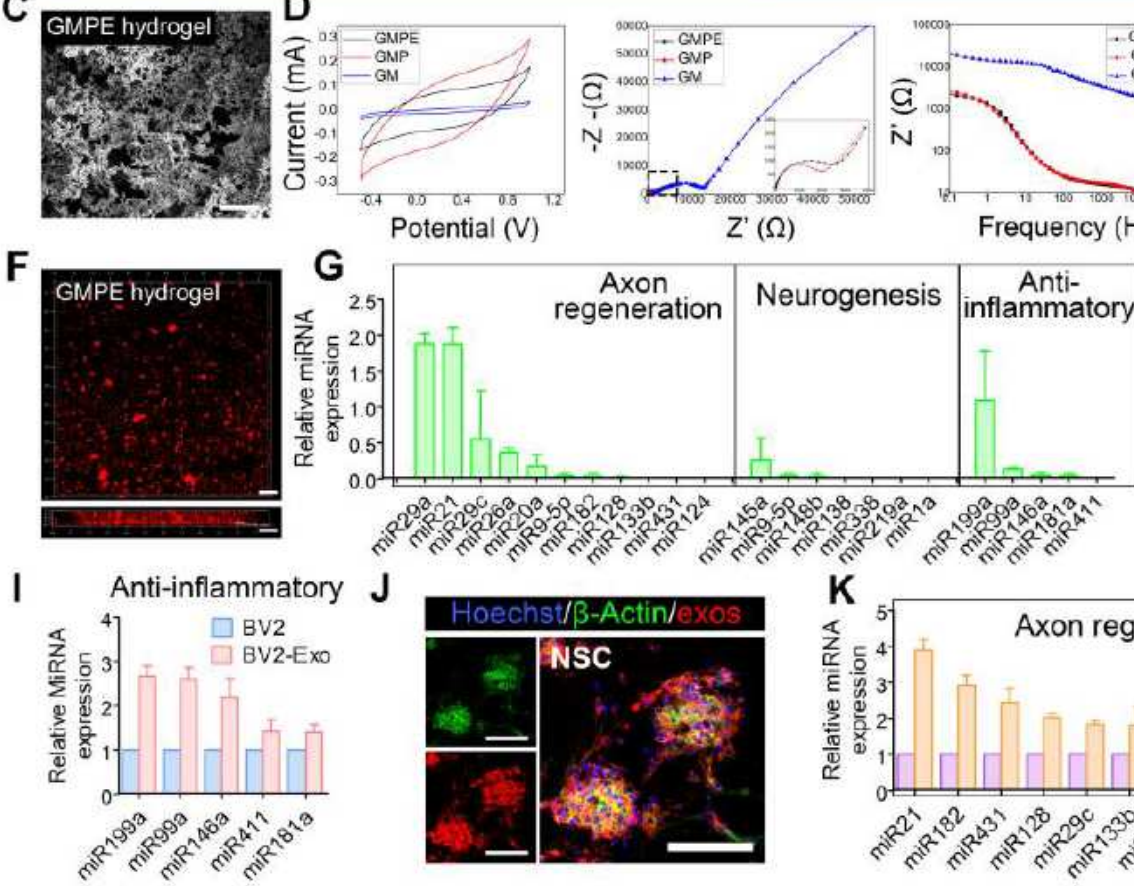

\section{J}

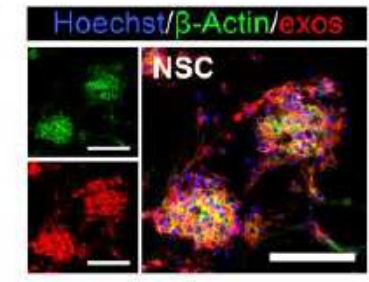

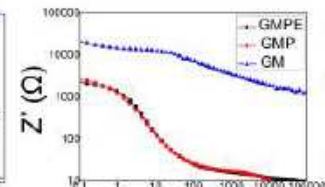

Frequency $(\mathrm{Hz})$

B

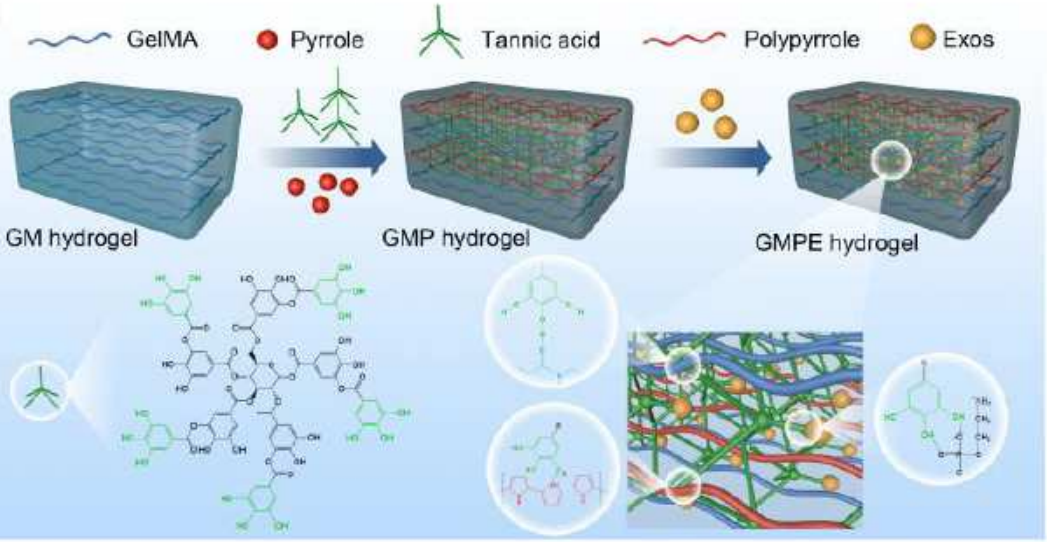

E

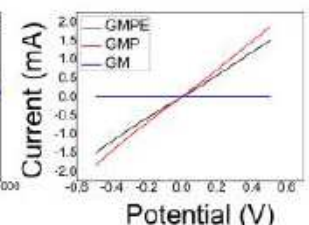

E

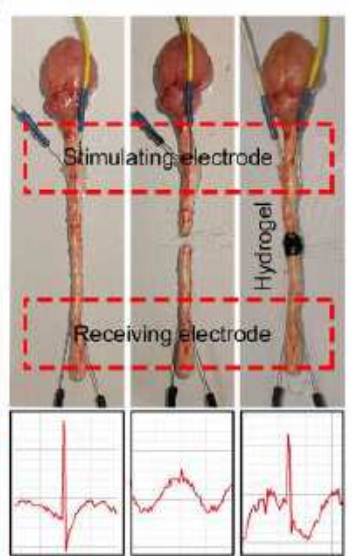

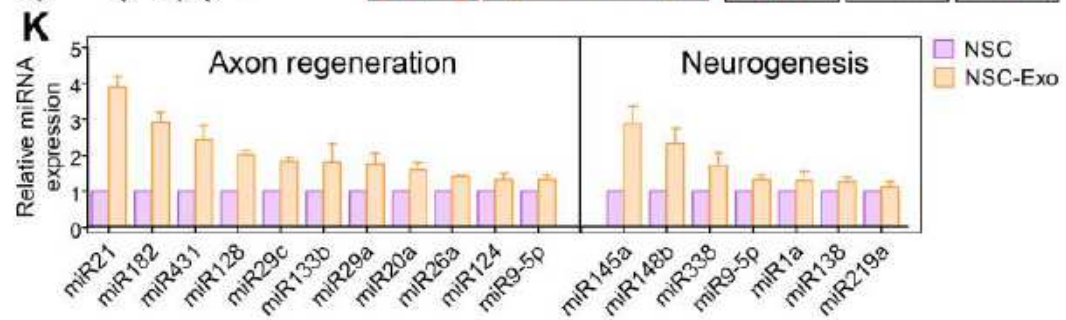

\section{Figure 1}

Characteristics of the GMPE hydrogels. (A) Illustration of how the GMPE hydrogel can reduce early inflammation, enhance neurogenesis and promote myelin-associated axonal regrowth to synergistically promote locomotor recovery after spinal cord hemisection. (B) The three-step synthesis procedure for the GMPE hydrogel was illustrated. The GMP hydrogel was synthesized by TA interacting with the amide bond on the GM backbone and the nitrogen groups on PPy chains. BMSC-exos were reversibly immobilized into GMP hydrogels via hydrogen bond formation between TA polyphenol groups and phosphate groups in exos phospholipid to form GMPE hydrogel. (C) Micro-structure of the GMPE hydrogel was observed by SEM. Scale bars, $25 \mu \mathrm{m}$. (D) Electrical characterization, including cyclic voltammograms (CV), electrochemical impedance (EIS), current-voltage (I-V) and Bode plots of GMP and GMPE hydrogels showed excellent electrical performance. (E) After the isolation of transected spinal cords, the stimulating electrical signals were retransmitted by GMPE hydrogels. (F) IF imaging showed 
that exos were evenly distributed into the GMPE hydrogel and the penetration depth of the exosomes was more than $100 \mu \mathrm{m}$. Scale bars, $100 \mu \mathrm{m}$. (G) RT-qPCR indicated that BMSC-exos express of axon regeneration-related, neurogenesis-related, and anti-inflammatory-related miRNAs $(n=3)$. (H) BV2 cells cultured on the GMPE hydrogel can normally phagocytize exos released from the hydrogel. Scale bars, $100 \mu \mathrm{m}$. (I) Anti-inflammatory-related miRNAs expression increased as the result of BV2 cells phagocytosing exos $(n=3)$. (J) PKH26 labeled exos were clearly detected in the cytoplasm of NSCs, suggesting successful in vitro endocytosis of exos released from the GMPE hydrogel. Scale bars, $100 \mu \mathrm{m}$. (K) Axon regeneration-related and neurogenesis-related miRNAs expression increased after NSCs phagocytize exos $(n=3)$.

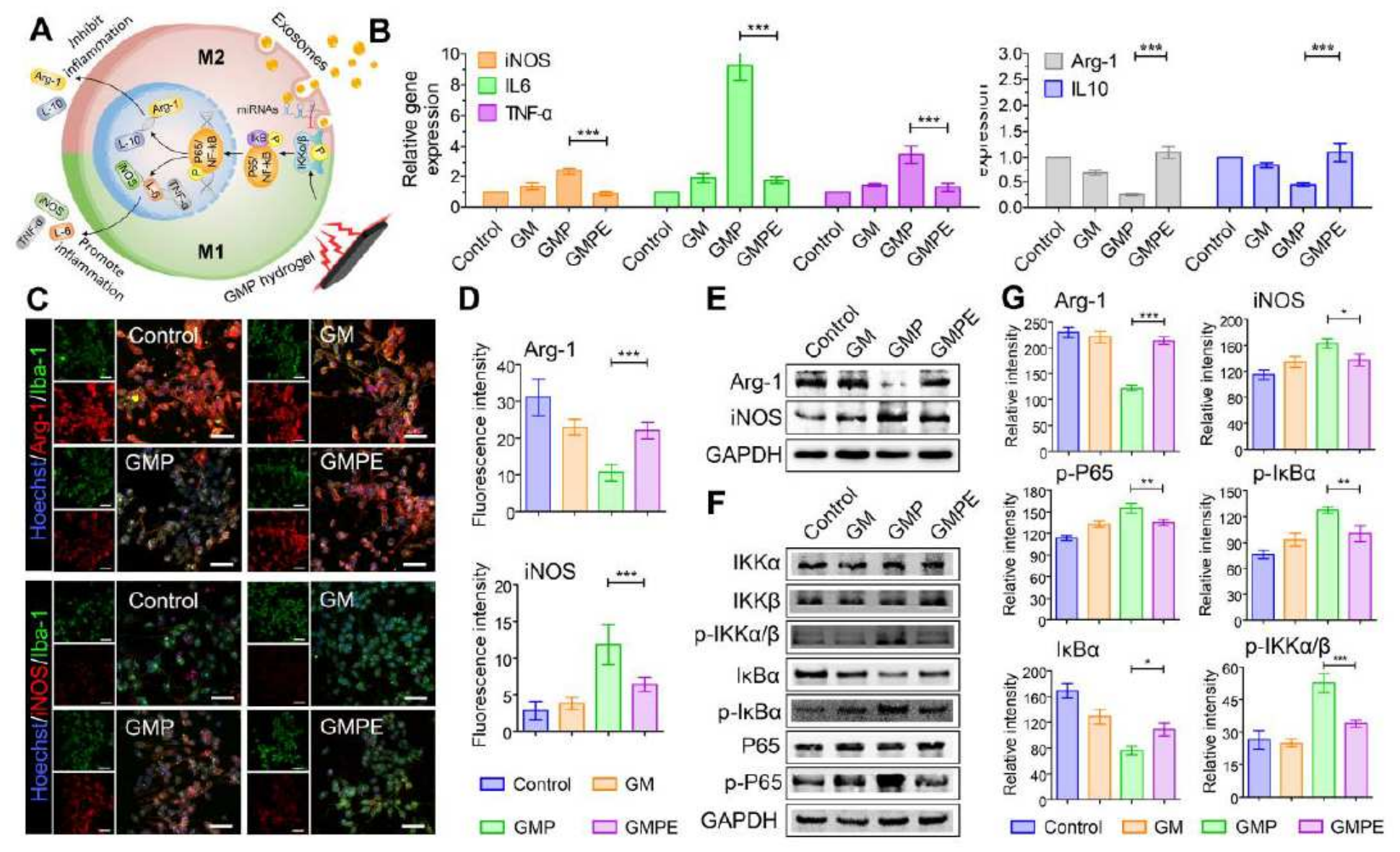

Figure 2

GMPE hydrogels promote microglia M1 to M2 switch by activating the NF-KB pathway. (A) An illustration of microglia switching from an M1- to M2-dominant phenotype through NF-kB pathway activity. (B) RTqPCR results of the level gene expression of pro-inflammatory factors Arg-1 and IL-10 and the antiinflammatory factors iNOS, IL-6 and TNF-a in BV2 cells cultured on hydrogels $(n=3)$. Statistical differences were determined using an ANOVA with Bonferroni's multiple comparison test. (C) IF imaging showing the proportion of Arg-1 positive and iNOS positive BV2 cells cultured in each hydrogel treatment group. Green IF represents the microglia/macrophage specific protein marker Iba-1, red fluorescence represents the M1/M2 microglia/macrophage phenotype marker iNOS or Arg-1, blue fluorescence represents the nuclear marker Hoechst 33342. Scale bars, $200 \mu \mathrm{m}$. (D) Quantification of fluorescence 
intensity of iNOS and Arg-1 level in each hydrogel treatment group $(n=5)$. Statistical differences were determined using ANOVA with Bonferroni's multiple comparison test. (E) GMPE hydrogel promoted BV2 cell M1 polarization. (F) GMPE hydrogel up-regulated the expression of Arg-1, iNOS, and relative proteins of NF-KB pathway, further indicating that the GMPE hydrogel promotes BV2 cell M1 polarization through NF-KB pathway activation. (G) Protein band intensity was quantified using Image $J(n=3)$. Statistical differences between gene expression and protein expression were determined using an ANOVA with Bonferroni's multiple comparison test $\left(* p<0.05,{ }^{* \star} p<0.01\right.$, $\left.{ }^{\star \star \star} p<0.001\right)$.

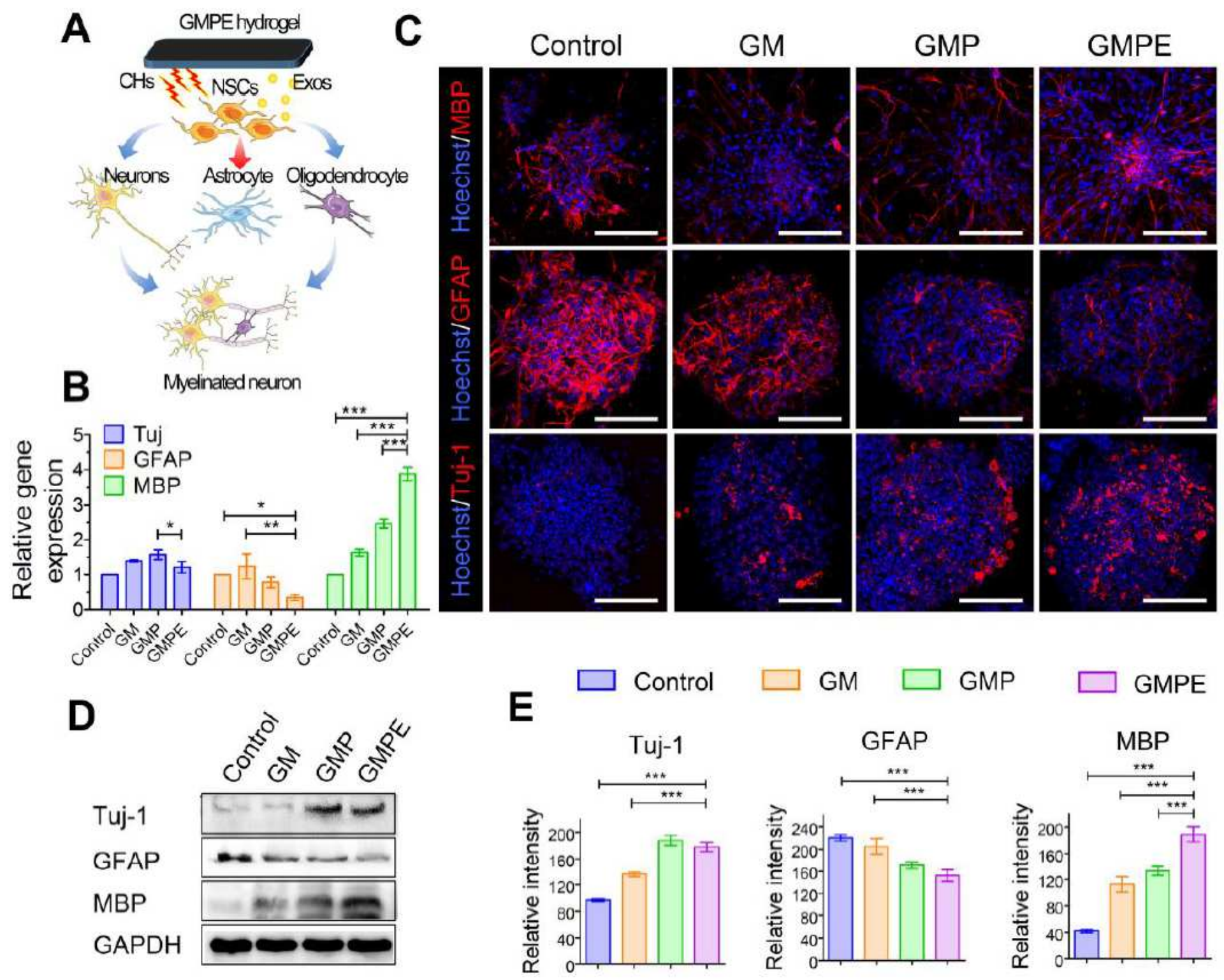

Figure 3

(A) An illustration showing that the GMPE hydrogel promotes NSC neuron and oligodendrocyte differentiation to form myelinated neurons, but inhibited astrocyte differentiation. (B) The RT-qPCR data show GMPE promote MBP expression $(n=3)$. Statistical differences were determined using ANOVA with Bonferroni's multiple comparison test. (C) IF imaging of NSC neuron, astrocyte, and oligodendrocyte differentiation after 7 days on control or hydrogel conditions. Red IF represents the neuron marker Tuj-1, 
astrocyte marker GFAP, or oligodendrocyte marker MBP, respectively. Scale bars, $100 \mu \mathrm{m}$. (D) WB analysis detected Tuj-1, GFAP, and MBP protein expression in NSCs cultured on hydrogels and control conditions for 7 days. (E) Protein band intensity was quantified $(n=3)$ and analyzed using an ANOVA with Bonferroni's multiple comparison test $\left({ }^{*} p<0.05\right.$, ${ }^{* *} p<0.01$, $\left.{ }^{* *} p<0.001\right)$.

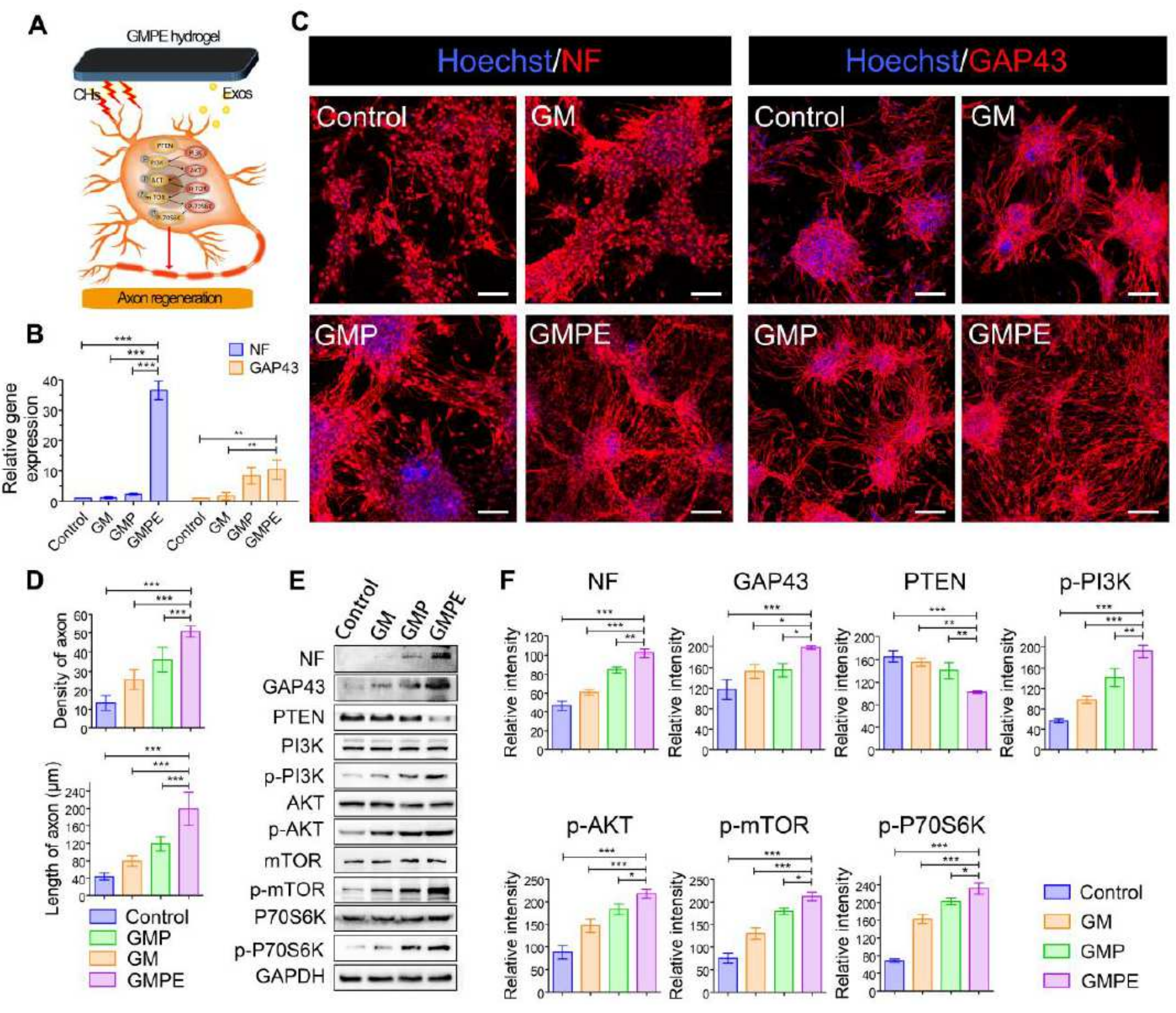

\section{Figure 4}

Axon outgrowth on hydrogels. (A) Illustration of the BMSC-exos conductive hydrogel mechanism and synergistic promotion of axon outgrowth through the activation of the PTEN/PI3K/AKT/mTOR pathway. (B) RT-qPCR indicating that GMPE hydrogels can promote NF and GAP43 gene expression $(n=3)$. Statistical differences determined using ANOVA with Bonferroni's multiple comparison test. (C) IF images of the axon-associated proteins NF and GAP43 in NSCs grown on hydrogels for 7 days. Red IF represents the NF or GAP43, respectively. Scale bars, $100 \mu \mathrm{m}$. (D) The density $(n=5)$ and length of axons were quantified using Image $J$ software $(n=11)$. Statistical differences were determined using an ANOVA with 
Bonferroni's multiple comparison test. (E) WB result of the expression of NF and GAP43 proteins and the relative protein expression of the PTEN/PI3K/AKT/mTOR pathway in NSCs cultured on control or hydrogel conditions for 7 days. (F) Protein band intensity was quantified $(n=3)$ and analyzed using an ANOVA with Bonferroni's multiple comparison test $(* p<0.05$, $* * p<0.01, * \star * p<0.001)$.

A
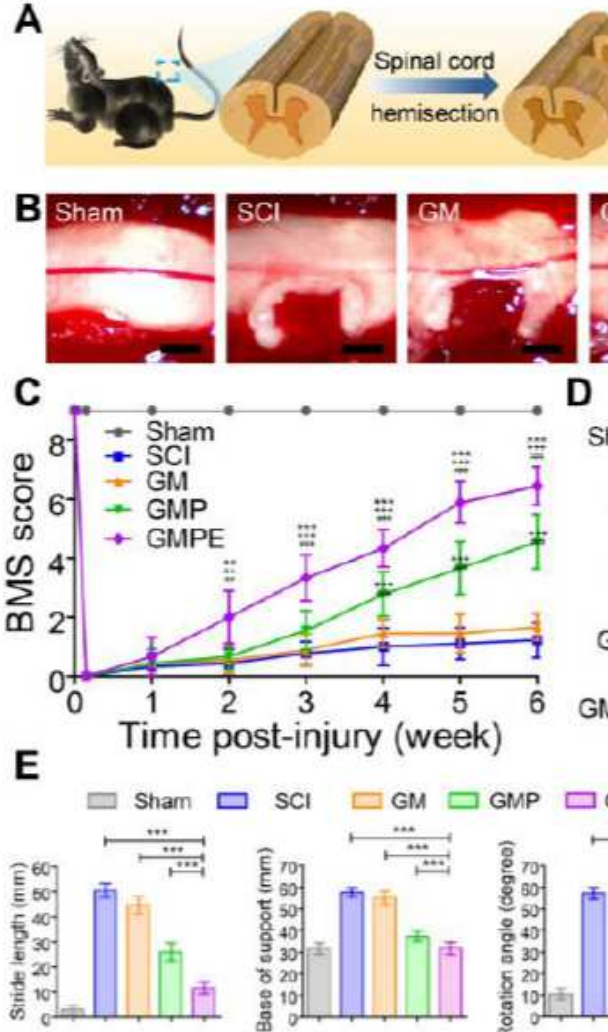

F Sham

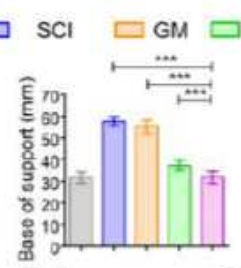

$\mathrm{SCl}$
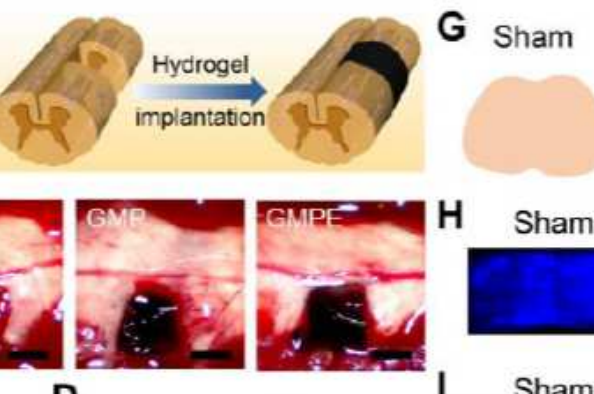

H Sham
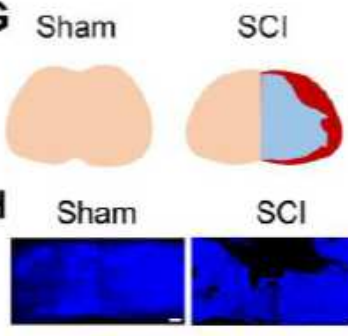

GM
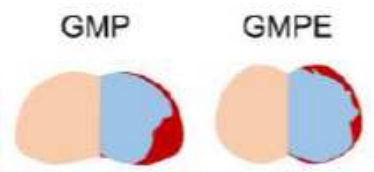

$\mathrm{SCl}$

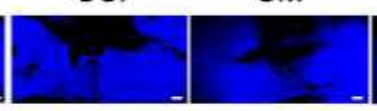

GMP

GMPE
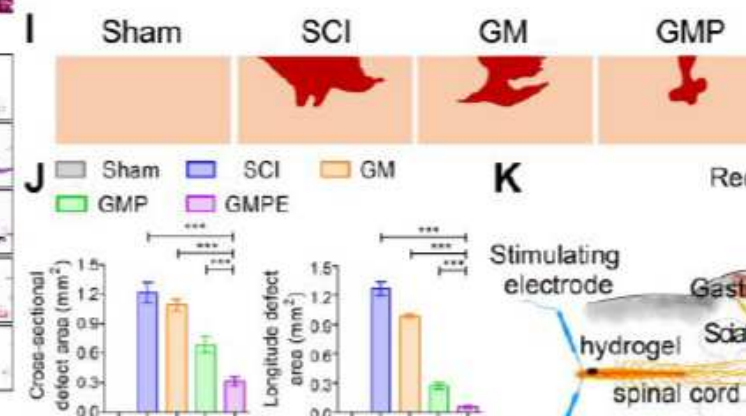

$\mathbf{K}$

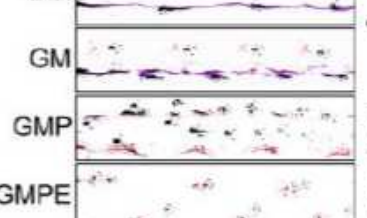

L
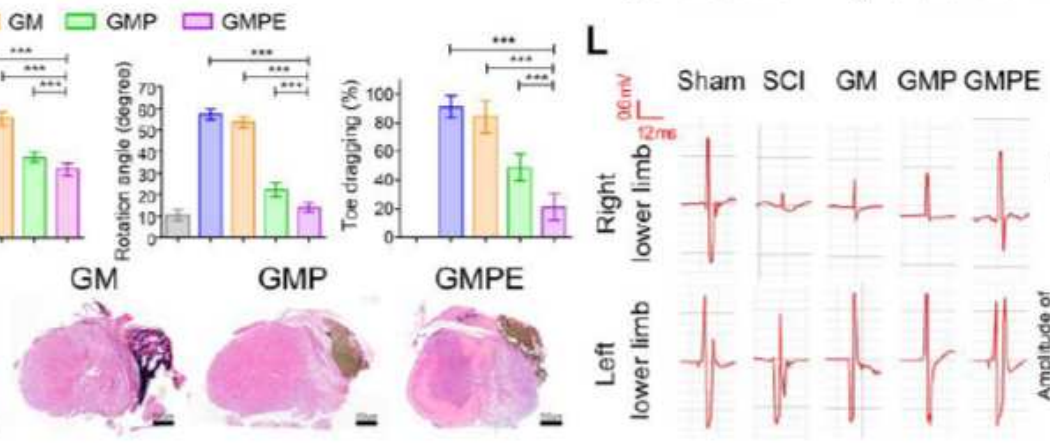

Sham SCl

GM GMP GMPE

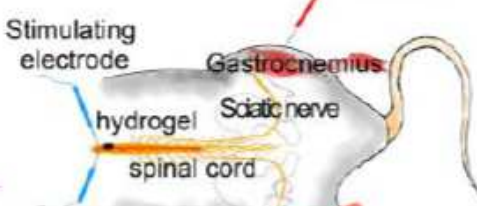

GMPE

M Sham $\square \mathrm{SCl} \square \mathrm{GM}$
$\square \mathrm{GMP} \square \mathrm{GMPE}$
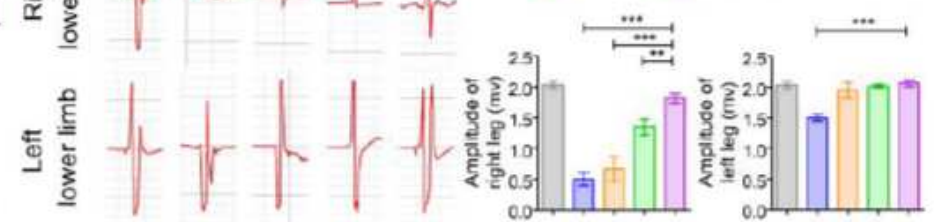

\section{Figure 5}

Functional recovery of mice in different groups. (A) Illustration of the spinal cord hemisection and hydrogel implantation. (B) Different hydrogels were implanted at the cavitary site. Scale bars, $1 \mathrm{~mm}$. (C) Right hindlimb locomotor recovery in mice was evaluated using BMS scoring on a grid. Mice treated with the GMPE hydrogel had improved BMS locomotor scores 2 weeks after injury $(n=9)$ when compared to GMP $\left({ }^{*} p<0.05, * \star p<0.01, * * * p<0.001\right)$ and GM $(+p<0.05,++p<0.01,+++p<0.001)$ hydrogels, and $\mathrm{SCl}(\#$ $p<0.05$, \#\# $p<0.01$, \#\#\# $p<0.001)$. Statistical differences were determined using an ANOVA with Bonferroni's multiple comparison test. (D) Representative footprints used to analyze recovery of hindlimb motor function. The forelimb footprints are shown in blue, and the hindlimb footprints in red. (E) Stride length, base of support, rotation angle and toe dragging were used to quantify the recovery of locomotion at 6 weeks after injury $(n=9)$. Statistical differences were determined using an ANOVA with Bonferroni's multiple comparison test. (F) HE staining showing the morphology of transverse spinal cord sections of sham and hydrogel implantation after SCI. (G) Representative reconstructions of the transverse spinal cord sections. Flesh-colored areas represent normal tissue, cavitary areas are highlighted in red, and blue- 
colored areas represent regenerated tissue. (H) IF images illustrating the morphology of longitudinal sections of the spinal cords. Scale bars, $200 \mu \mathrm{m}$. (I) Representative images of reconstructed spinal cord longitudinal section. Flesh-colored areas represent normal tissue, while red-colored areas represent the cavitary areas. (J) Cavity volume of transverse spinal cord and longitudinal sections was quantified $(n=3)$. Statistical differences were determined using an ANOVA with Bonferroni's multiple comparison test. $(\mathrm{K})$ Illustration of the CMAP testing protocol in mice from GMPE hydrogel treatment group. (L) CMAP results from normal mice and those in different hydrogel implantation groups 6 weeks post-treatment. (M) Quantification of the CMAP amplitudes measured in mice from sham and hydrogel treatment groups $(n=3)$. Statistical differences were determined using an ANOVA with Bonferroni's multiple comparison test $(* p<0.05, * * p<0.01, * * * p<0.001)$.

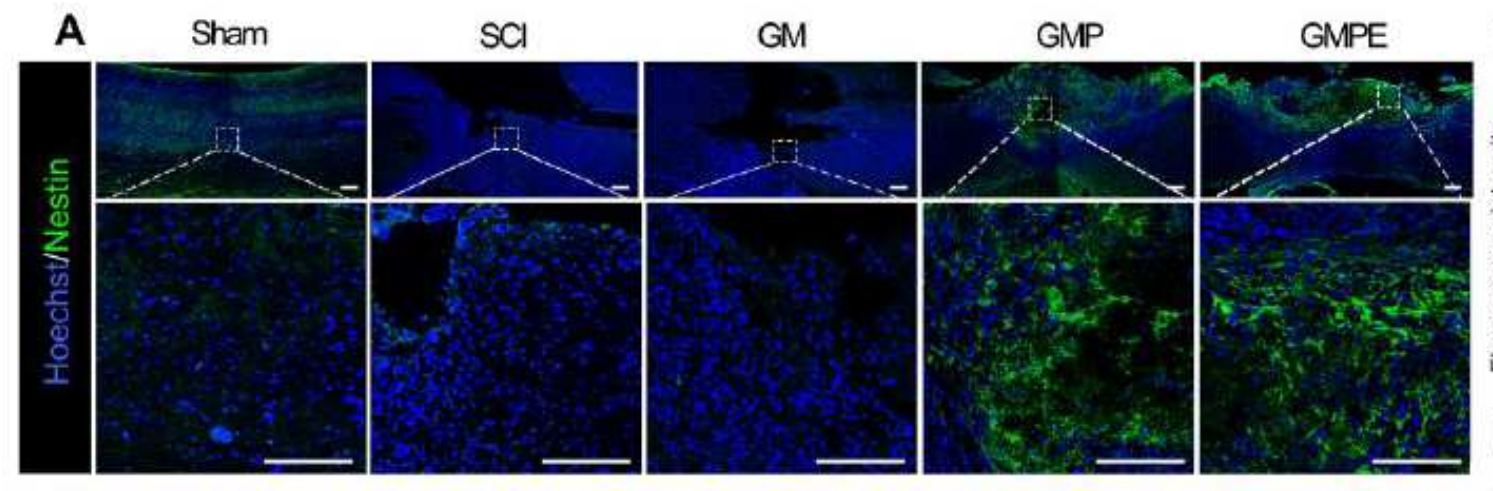

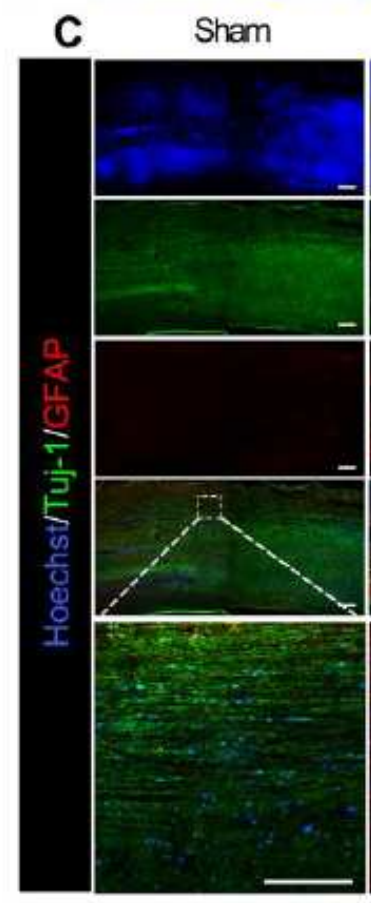
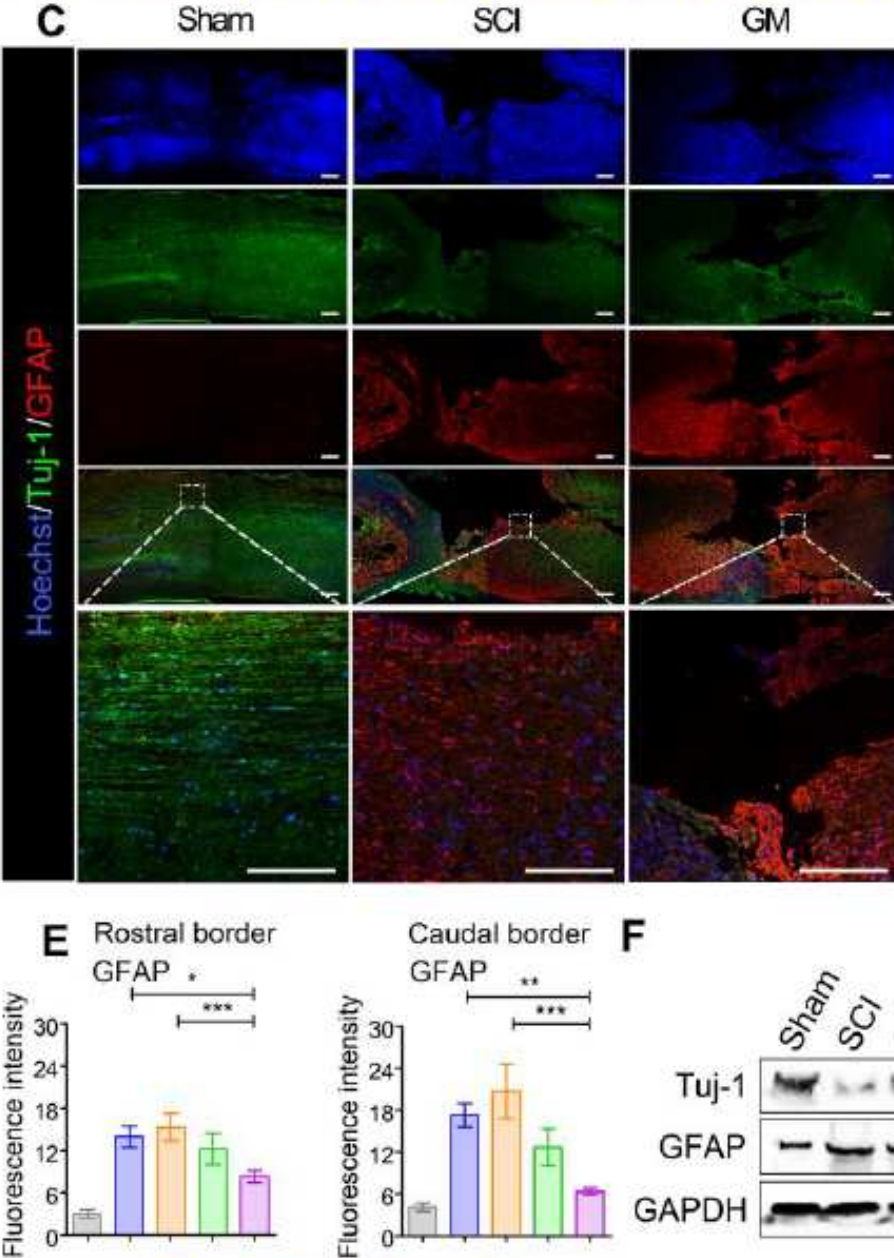

$\square$ Sham $\square \mathrm{SCl} \square \mathrm{GM} \square \mathrm{GMP} \square \mathrm{GMPE}$
$\mathbf{F}$
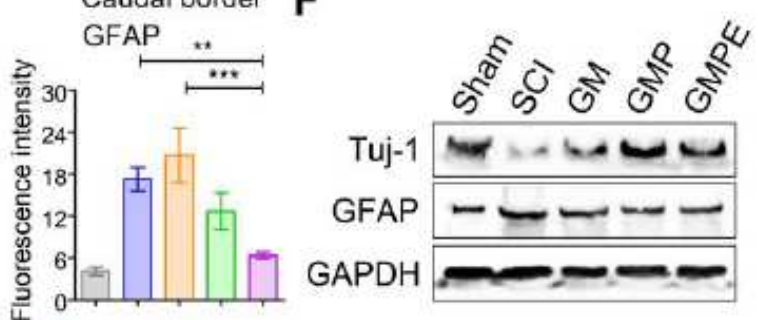

G Tuj-1

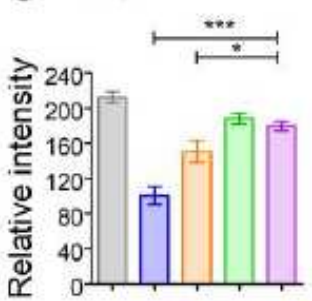

$\square$ Sham $\square \mathrm{SCl} \square \mathrm{GM} \square \mathrm{GMP} \square \mathrm{GMPE}$
B Nestin

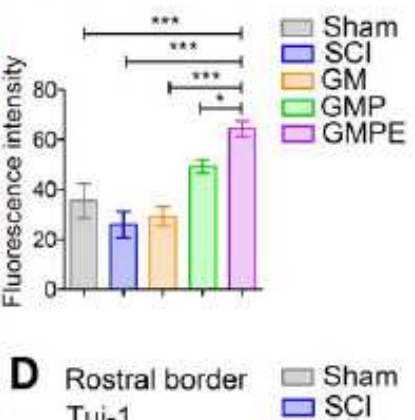
$\square \mathrm{GM}$ $\square$ GMP $\square$ GMPE 


\section{Figure 6}

GMPE hydrogel implantation promotes endogenous neurogenesis. (A) Nestin-positive endogenous NSCs spontaneously migrated into the GMPE hydrogel 6 weeks after SCI. Green IF represents the NSC marker nestin. Scale bars, $200 \mu \mathrm{m}$. (B) Graph of the quantification of nestin IF intensity 6 weeks after SCl. The region of interest $(\mathrm{ROI})$ was the injury site center $(\mathrm{n}=3)$. Statistical differences were determined using an ANOVA with Bonferroni's multiple comparison test. (C) The GMPE hydrogel promotes endogenous NSC neuronal differentiation while inhibiting astrocytic differentiation in vivo. Green IF represents the neuronal marker Tuj-1. Red IF represents the astrocytic marker GFAP. Scale bars, $200 \mu \mathrm{m}$. (D) Graph of the Tuj-1 IF intensity quantification. The fluorescence intensity was measured at three ROls: the rostral and caudal borders, and the injury site center $(n=3)$. Statistical differences were determined using an ANOVA with Bonferroni's multiple comparison test. (E) Graph of GFAP IF intensity quantification at same timepoint at the ROls: rostral and caudal borders of the injury site $(n=3)$. Statistical differences were determined using an ANOVA with Bonferroni's multiple comparison test. (F) WB analysis of the neuronal and astrocytic differentiation protein markers in each treatment group. (G) Quantitative measurement of protein band intensity included calculating the statistical differences between samples $(n=3)$ using ANOVA with Bonferroni's multiple comparison test ( ${ }^{\star} p<0.05,{ }^{* \star} p<0.01$, $\left.* \star \star * 0.001\right)$. 


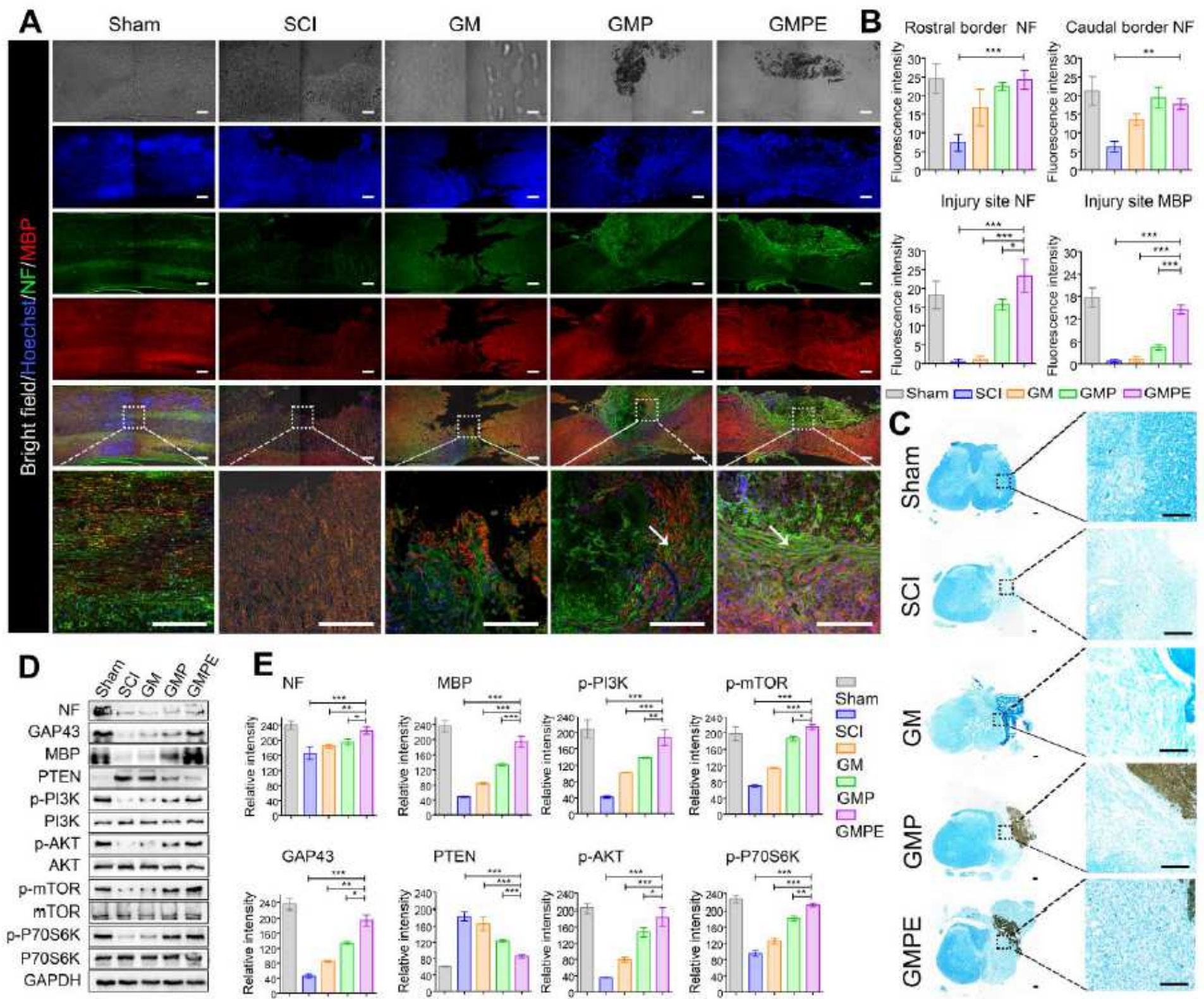

\section{Figure 7}

GMPE hydrogel implantation promotes axon regeneration and remyelination in vivo. (A) Axonal regeneration and remyelination was evaluated in the five treatment groups 6 weeks post operation using IF imaging. Green IF represents the NF axon marker. Red IF represents the MBP oligodendrocyte marker. Scale bars, $200 \mu \mathrm{m}$. (B) Graph of NF and MBP IF intensity quantified from ROls at both ends and the center of the injury site $(n=3)$. GFAP IF intensity ROI were measured from center of the injury site $(n=3)$. Statistical differences were determined using an ANOVA with Bonferroni's multiple comparison test. (C) Remyelination was evaluated by LFB staining which showed that nerve myelin was detected around the GMPE hydrogel. Scale bars, $200 \mu \mathrm{m}$. (D) Protein expression of NF, GAP43, MBP, and PTEN/PI3K/AKT/mTOR pathway proteins; GADPH was included as an expression control. (E) Graph of protein band intensity quantification $(n=3)$. Statistical differences were determined using an ANOVA with Bonferroni's multiple comparison test $(* p<0.05$, ** $p<0.01, * * * p<0.001)$. 


\section{Supplementary Files}

This is a list of supplementary files associated with this preprint. Click to download.

- Supplementaryinformation.pdf 